\title{
Contact Geometry of Curves
}

\author{
Peter J. VASSILIOU
}

Faculty of Information Sciences and Engineering, University of Canberra, 2601 Australia

E-mail: peter.vassiliou@canberra.edu.au

Received May 07, 2009, in final form October 16, 2009; Published online October 19, 2009

doi:10.3842/SIGMA.2009.098

\begin{abstract}
Cartan's method of moving frames is briefly recalled in the context of immersed curves in the homogeneous space of a Lie group $G$. The contact geometry of curves in low dimensional equi-affine geometry is then made explicit. This delivers the complete set of invariant data which solves the $G$-equivalence problem via a straightforward procedure, and which is, in some sense a supplement to the equivariant method of Fels and Olver. Next, the contact geometry of curves in general Riemannian manifolds $(M, g)$ is described. For the special case in which the isometries of $(M, g)$ act transitively, it is shown that the contact geometry provides an explicit algorithmic construction of the differential invariants for curves in $M$. The inputs required for the construction consist only of the metric $g$ and a parametrisation of structure group $S O(n)$; the group action is not required and no integration is involved. To illustrate the algorithm we explicitly construct complete sets of differential invariants for curves in the Poincaré half-space $H^{3}$ and in a family of constant curvature 3-metrics. It is conjectured that similar results are possible in other Cartan geometries.
\end{abstract}

Key words: moving frames; Goursat normal forms; curves; Riemannian manifolds

2000 Mathematics Subject Classification: 53A35; 53A55; 58A15; 58A20; 58A30

\section{Introduction}

The classical topic of immersed submanifolds in homogeneous spaces via repère mobile or moving frames is discussed here in the simplest case, that of curves. Several authors have written on the method of repère mobile, over the years since Cartan's works, such as [5]; these include S.S. Chern [6], J. Favard [8], P.A. Griffiths [12], G.R. Jensen [14], M.L. Green [11], R. Sulanke [26], R. Sharpe [21] and M.E. Fels \& P.J. Olver [9, 10]. Some of these authors have the goal of placing Cartan's method on a firm theoretical foundation as well as extending its range of application beyond the classical realm. More recently, a reformulation of the method of moving frames, due to Fels and Olver $[9,10]$ has lead to renewed activity and a great many new applications and perspectives, have arisen (see [18] and references therein). Whereas Cartan emphasised the construction of canonical Pfaffian systems whose integral manifolds are the Frenet frames along the submanifold, a much more direct approach is favoured in the Fels-Olver formulation and this has a number of significant advantages. However, in this paper, we shall reconsider the role of Pfaffian systems in the method of moving frames in the light of recent results in the geometry of jet spaces with the principal goal of making the contact geometry of curves more explicit and giving some indication about its possible applications. Another goal is to provide additional insight into the relationship between Cartan's method of moving frames and the equivariant method of Fels and Olver ${ }^{1}$.

\footnotetext{
*This paper is a contribution to the Special Issue "Élie Cartan and Differential Geometry". The full collection is available at http://www.emis.de/journals/SIGMA/Cartan.html

${ }^{1} \mathrm{~A}$ point we make herein is that the geometry of jet spaces provides a useful mediation between the two approaches.
} 
The considerations in this paper were inspired by a paper of Shadwick and Sluis [20], in which the authors observed that many of the Pfaffian systems derived by Cartan admit a Cartan prolongation which is locally diffeomorphic to the contact distribution on jet space $J^{k}\left(\mathbb{R}, \mathbb{R}^{q}\right)$, for some $k$ and $q$, thereby explicitly adding contact geometry to Cartan's method of moving frames. Another way to view the aims of this paper is the further development of the ideas in [20] in relation to moving frames for curves by exploring the application of a recent generalisation [27, 28] of the Goursat normal form from the theory of exterior differential systems [25, 3] allowing for the explicit determination of differential invariants and other invariant data in cases which have not been previously explored in detail. Of particular interest are curves in general Cartan geometries and in this paper we have focused on the Riemannian case and conjecture that similar results hold for other Cartan geometries.

We show that given any $n$-dimensional Riemannian manifold $\left(M^{n}, g\right)$ then the Pfaffian system whose integral manifolds determine the Frenet frames along curves in $M$ has a Cartan prolongation which can be identified with the contact system on jet space $J^{n}\left(\mathbb{R}, \mathbb{R}^{n-1}\right)$. The explicit construction of the identification requires only differentiation. In case the isometries of $\left(M^{n}, g\right)$ act transitively then the construction of the differential invariants that settles the equivalence problem for curves up to an isometry differs from the approach of FelsOlver in that explicit a priori knowledge of the isometries or even the infinitesimal isometries is not required; as in the Fels-Olver method no integration is called for. The inputs for algorithm Riemannian curves consist only of the metric $g$ and a realisation of the Lie group $S O(n)$.

Moreover, a contention of this paper is that the contact geometry of submanifolds to be described below should be a fundamental fact and lead to useful points of view that complement and enhance the geometric analysis of submanifolds by existing methods such as Cartan's method of moving frames and the equivariant moving frames method of Fels and Olver.

The content of this paper is as follows. After briefly recalling the method of moving frames, as practiced by Cartan, we study one of the simplest non-trivial examples: curves in 2-dimensional equi-affine geometry. It is then shown how the (classical) Goursat normal form applies to give the unique differential invariant and moving frame, explicitly. This familiar, illustrative example encapsulates the ideas proposed in this paper and is simple enough so that all details can be given. An account of the generalised Goursat normal form [27, 28] is then given in the special case of total prolongations (uniform Goursat bundles) in preparation for the study of immersed curves in higher dimensional Cartan geometries. Section 4 illustrates the principles developed in the previous section by applying it to study curves in 3d-equi-affine geometry, computing the complete set of differential invariants via the generalised Goursat normal form.

Section 5 is devoted to the contact geometry of curves in any Riemannian manifold and contains the main application of the paper. The general method is used to explicitly derive the differential invariants for curves in the Poincaré half-space $H^{3}$ and for curves in a family of constant curvature 3-metrics. These invariants do not seem to have appeared in the literature before. The results demonstrate that the contact geometry of submanifolds can offer an alternative path to invariant data for curves besides the Fels-Olver equivariant method and Cartan's method which, in the latter case, relies so much on geometric insight and special tricks for the construction of the Frenet frames ${ }^{2}$.

Finally, it should be mentioned that while this paper only explores the case of curves, the contact geometry of higher dimensional submanifolds could be similarly studied, commencing with the well known characterisation of contact systems in any jet space given in $[2,30]$.

\footnotetext{
${ }^{2}$ In Cartan's writings the distinction between the Frenet frames along a submanifold and the exterior differential system whose solutions are the Frenet frames is sometimes blurred. It's best to keep these two notions quite separate since the construction of the latter is algorithmic while that of the former is not.
} 


\section{Method of repère mobile applied to curves}

According to [14] the general problem treated by Cartan in [5] and elsewhere is that of the invariants of submanifolds in the homogeneous space of a Lie group $G$ under the action of $G$. In this section, I will give a very brief description of the method of repère mobile, Cartan's principal tool for addressing this type of problem. More complete discussions can be found in the references quoted above such as $[8,12,14,26]$. The exposition given by Cartan in [4] is still well worth reading.

Let $G$ be a Lie group and $H \subset G$ a closed subgroup. Then we have the $H$-principal bundle

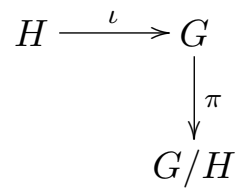

of left cosets of $H$ in $G$ and we let $M:=G / H$. Map $\pi$ is the natural projection assigning a leftcoset $g H$ to each element of $g \in G$. There is a natural left-action of $G$ on $M: g \cdot z H=g z H$, for all $g \in G$. Let $x$ be a local coordinate system on $M$. Cartan typically began with a representation of $G$ which could be "decomposed" into columns $e_{1}, e_{2}, \ldots, e_{r}$ of $H \subset G$ and $\boldsymbol{x}$, a column vector whose components are the coordinates $x$ on $M$.

Cartan defines differential 1-forms $\omega^{i}, 1 \leq i \leq r$ by

$$
d \boldsymbol{x}=\sum_{i=1}^{r} \omega^{i} \otimes e_{i} .
$$

The 1-forms $\omega^{i}$ are semi-basic for $\pi$. Furthermore, we have 1-forms $\omega_{i}^{j}$ defined by

$$
d e_{i}=\sum_{j=1}^{r} \omega_{i}^{j} \otimes e_{j}
$$

The 1 -forms $\omega^{i}, \omega_{i}^{j}, i, j=1, \ldots, r$ are the components of the Maurer-Cartan form $\omega$ on $G$; the integral submanifolds of the Pfaffian system

$$
\omega^{1}=0, \quad \omega^{2}=0, \ldots, \quad \omega^{r}=0
$$

foliates $G$ by the left cosets of $H$.

Suppose $f: T \rightarrow M$ is an immersion of a manifold $T$ into $M$. Then a moving frame is a local map $F: T \rightarrow G$ such that $f=\pi \circ F$. That is, the moving frame assigns to each point $t \in T$ a coset $f(t) \in G / H$. With this general set up, Cartan addresses the following problem for submanifolds of $M$. Let $f_{1}: T_{1} \rightarrow M$ and $f_{2}: T_{2} \rightarrow M$ be submanifolds. Find necessary and sufficient conditions, in the form of differential invariants, such that there is a local diffeomorphism $\mu: T_{1} \rightarrow T_{2}$ and element $g \in G$ such that

$$
f_{2} \circ \mu=g \cdot f_{1} .
$$

The 'o' denotes function composition while '.' continues to denote the left-action of $G$ on $M$. A special case of this is the so-called fixed parametrisation problem where one takes $T_{1}=T_{2}=T$ and $\mu$ is the identity on $T$. This congruence problem is the one that will be studied in this paper.

In case the submanifolds of $M$ are curves, Cartan begins by choosing a codimension 1 subset of the semibasic 1-forms and defines the Pfaffian system

$$
\Omega: \omega^{2}=0, \quad \omega^{3}=0, \ldots, \quad \omega^{r}=0 .
$$


One studies the solutions of $\Omega$ since these project via $\pi$ down to curves in $G / H$, which are the objects of interest. One way to do this is via the Cartan-Kähler theorem [3]. Accordingly, one computes the exterior derivatives of the $\omega^{j}, j=2, \ldots, r$ and appends these "integrability conditions" to $\Omega$ forming the differential ideal $\bar{\Omega}$ with independence form $\omega^{1}$. This procedure allows one to prove existence of integral curves for $\Omega$ and provides information about the number of such integral curves. However, this makes no use of the special origin of the 1 -forms in $\Omega$, arising as they do from the Maurer-Cartan form $\omega$ on $G$. As a result of this one can go much further. From the structure equations of $\omega$ and the vanishing of the exterior derivatives $d \omega^{i}$ we deduce additional 1-form equations of the form

$$
\omega_{i}^{j}-p_{i}^{j} \omega^{1}=0
$$

for some functions $p_{i}^{j}$ on $G$, which are appended to $\Omega$ as integrability conditions, thereby forming the new Pfaffian system

$$
\bar{\Omega}: \omega^{2}=0, \quad \omega^{3}=0, \quad \ldots, \quad \omega^{r}=0, \quad \omega_{i}^{j}-p_{i}^{j} \omega^{1}=0 .
$$

In essence, the method of moving frames consists of using the fact that $H$ acts on the fibres of $G \rightarrow G / H$ on the right inducing a transformation of the Maurer-Cartan form $\omega$ on $G$, [23, Chapter 7]. Indeed, the transformation

$$
\left(\boldsymbol{x}, e_{1}, \ldots, e_{r}\right) \mapsto\left(\boldsymbol{x}, e_{1}, \ldots, e_{r}\right) h, \quad \forall h \in H
$$

on $G$ induces the transformation

$$
\omega \mapsto \operatorname{Ad}\left(h^{-1}\right) \omega+h^{-1} d h=\widetilde{\omega}
$$

on the Maurer-Cartan form $\omega$. In turn, this induces a transformation on the functions $p_{i}^{j}$. To proceed further we recall the notion of a Cartan prolongation.

Definition 1. Let $\mathcal{I}$ be a Pfaffian system on manifold $M$ and $\mathfrak{p}: \widehat{M} \rightarrow M$ a fibre bundle. A Pfaffian system $\widehat{\mathcal{I}}$ on $\widehat{M}$ is said to be a Cartan prolongation of $(M, \mathcal{I})$ if

1) $\mathfrak{p}^{*} \mathcal{I} \subseteq \widehat{\mathcal{I}}$

2) for every integral submanifold $\sigma: S \rightarrow M$ of $\mathcal{I}$ there is a unique integral submanifold $\widehat{\sigma}: S \rightarrow \widehat{M}$ of $\widehat{\mathcal{I}}$ that projects to $\sigma ;$ that is, $\sigma=\mathfrak{p} \circ \widehat{\sigma}$.

We say that $\widehat{\sigma}$ is the Cartan lift of $\sigma$.

If we choose to view $\left(G \times \mathbb{R}^{s}, \bar{\Omega}\right)$, where the factor $\mathbb{R}^{s}$ carries the "parameters" $p_{i}^{j}$, as a Cartan prolongation of $(G, \Omega)$ then (3) induces a reduction of the trivial bundle $G \times \mathbb{R}^{s} \rightarrow G$ by normalising the coordinates $p_{i}^{j}$ on the fibres to simple constants like 0 and \pm 1 .

Once $\bar{\Omega}$ has been normalised, the process begins again by taking exterior derivatives of the enlarged, normalised Pfaffian system arising from $\bar{\Omega}$. Each step selects a subgroup $K \subset H$. If the process terminates at $K=\{$ identity $\}$ of $G$ then the resulting Pfaffian system arising from $\bar{\Omega}$ is canonical. The integral submanifolds of $\bar{\Omega}$ are the Frenet frames, $\mathfrak{F}$. Hereafter we shall label this canonical Pfaffian system by the symbol $\Omega_{\mathfrak{F}}$.

The main assertion made in this paper is that the canonical Pfaffian system $\Omega_{\mathfrak{F}}$ determining each Frenet frame along an immersed curve admits a Cartan prolongation $\widehat{\Omega}_{\mathfrak{F}}$ on $E:=G \times \mathbb{R}^{\nu}$ for some $\nu$, so that $\left(E, \widehat{\Omega}_{\mathfrak{F}}\right)$ is locally diffeomorphic to a jet space $\left(J^{k}\left(\mathbb{R}, \mathbb{R}^{q}\right), \Omega^{k}\left(\mathbb{R}, \mathbb{R}^{q}\right)\right)$ where $\Omega^{k}\left(\mathbb{R}, \mathbb{R}^{q}\right) \subset T^{*} J^{k}\left(\mathbb{R}, \mathbb{R}^{q}\right)$ is the contact sub-bundle. The coordinates on the $\mathbb{R}^{\nu}$ factor of $E$ 
carry the differential invariants of the problem. Indeed, the integral manifolds of $\widehat{\Omega}_{\mathfrak{F}}$, say, $\widehat{\Gamma}: I \rightarrow G \times \mathbb{R}^{\nu}$ project down to the Frenet lifts $\Gamma$ of curves $\gamma: I \rightarrow M$ as in

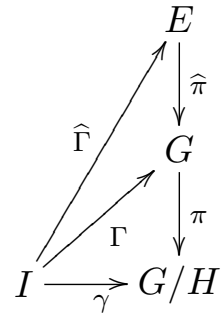

where $I \subseteq \mathbb{R}$ is an interval. Hereafter, one of our goals is to give examples which demonstrate the assertion made above, namely that the Pfaffian system $\widehat{\Omega}_{\mathfrak{F}}$ can be identified with a contact system. This identification can be constructed explicitly and provides explicit coordinate formulas for all the invariant data: differential invariants, Fels-Olver equivariant moving frames and invariant differential forms. In Section 5 we will prove that this procedure can be applied to curves in any Riemannian manifold and in that case it is algorithmic ${ }^{3}$. Importantly, one is not required to explicitly know the group action a priori. Before this we will work out some pedagogical examples. The first of these is sufficiently low dimensional so that all details can be given.

\subsection{Curves in the equi-affine plane}

The goal in this subsection is to provide a simple illustration of the method of moving frames as described in the previous subsection. We will construct the Frenet frame $\mathfrak{F}$ for a plane curve up to equi-affine transformations by constructing the canonical Pfaffian system $\Omega_{\mathfrak{F}}$ and the appropriate Cartan prolongation $\widehat{\Omega}_{\mathfrak{F}}$, as described above. Here equi-affine transformations means the standard transitive action of the Lie group $G=S L(n, \mathbb{R}) \ltimes \mathbb{R}^{n}$ on $\mathbb{R}^{n}$. For plane curves we take $n=2$; the action on $\mathbb{R}^{2}$ with local coordinates $\xi_{1}, \xi_{2}$ is

$$
\left[\begin{array}{l}
\xi_{1} \\
\xi_{2}
\end{array}\right] \mapsto A\left[\begin{array}{l}
\xi_{1} \\
\xi_{2}
\end{array}\right]+\left[\begin{array}{l}
x \\
y
\end{array}\right]
$$

where $A \in S L(2, \mathbb{R})$ and $x, y \in \mathbb{R}$. We identify $\mathbb{R}^{2}$ with $G / S L(2, \mathbb{R})$ where the elements of $G$ are matrices of the form

$$
g=\left[\begin{array}{lll}
1 & 0 & 0 \\
x & a & b \\
y & c & d
\end{array}\right]
$$

and $a d-b c=1$. We call this homogeneous space the affine plane and denote it by $\mathbb{A}^{2}$. For local coordinates on $\mathbb{A}^{2}$ we take $\boldsymbol{x}$, the first column of $g \in G$ and $e_{1}, e_{2}$ are the next two columns of $g$. Equations (1), (2) give the semi-basic forms

$$
\omega^{1}=\beta d x-b d y, \quad \omega^{2}=-c d x+a d y,
$$

and connection forms

$$
\omega_{1}^{1}=\beta d a-b d c, \quad \omega_{2}^{1}=\frac{1}{a}\left(\beta b d a+d b-b^{2} d c\right), \quad \omega_{1}^{2}=a d c-c d a,
$$

\footnotetext{
${ }^{3}$ In this paper a construction or procedure is said to be algorithmic if it can be performed only by differentiation and "algebraic operations", which includes constructing the inverse of a local diffeomorphism. However, integration is strickly excluded.
} 
where $\beta=(1+b c) / a$, where we have chosen a chart on $S L(2, \mathbb{R})$ in which $a \neq 0$; note that $\omega_{1}^{1}+\omega_{2}^{2}=0$. It is useful to record the structure equations

$$
\begin{aligned}
& d \omega^{1}=\omega^{1} \wedge \omega_{1}^{1}+\omega^{2} \wedge \omega_{2}^{1}, \\
& d \omega^{2}=\omega^{1} \wedge \omega_{1}^{2}-\omega^{2} \wedge \omega_{1}^{1}, \\
& d \omega_{1}^{1}=\omega_{1}^{2} \wedge \omega_{2}^{1}, \\
& d \omega_{2}^{1}=-2 \omega_{1}^{1} \wedge \omega_{2}^{1}, \\
& d \omega_{1}^{2}=2 \omega_{1}^{1} \wedge \omega_{1}^{2} .
\end{aligned}
$$

Successive adapted frames are integral curves of certain Pfaffian systems which will be denoted by $\Omega^{i}, i=1,2, \ldots$. The first adapted frames for curves in $\mathbb{A}^{2}$ are integral curves of the Pfaffian system $\Omega^{1}$, consisting of the single 1 -form equation

$$
\Omega^{1}: \omega^{2}=0
$$

with independence form $\omega^{1}$. From structure equations (6), we obtain $0=d \omega^{2} \equiv \omega^{1} \wedge \omega_{1}^{2}$ mod $\omega^{2}$ and hence to complete $\Omega^{1}$ to a differential ideal $\bar{\Omega}^{1}$ we extend it by appending the 2 -form equation $\omega_{1}^{2} \wedge \omega^{1}=0$. This equation implies that there is a function $p$ on $G$ such that the 2 -form equation can be replaced by $\omega_{1}^{2}-p \omega^{1}=0$, a kind of "first integral". We extend $\Omega^{1}$ by this 1 -form equation and rename the extended Pfaffian system $\bar{\Omega}^{1}$ to get

$$
\bar{\Omega}^{1}: \omega^{2}=0, \quad \omega_{1}^{2}-p \omega^{1}=0 .
$$

Note that the reconstituted $\bar{\Omega}^{1}$ is no longer a differential ideal.

As discussed in Section 2, an element $h \in H$ acts on the frame $\left[\boldsymbol{x}, e_{1}, e_{2}\right]$ over each point $\boldsymbol{x} \in G / H$ on the right inducing the transformation (4) on the Maurer-Cartan form on $G$. This, in turn induces a transformation on the function $p$. The subgroup $H_{1} \subset H$ that leaves $\Omega^{1}$-invariant has representation

$$
\left[\begin{array}{ccc}
1 & 0 & 0 \\
0 & a & b \\
0 & 0 & 1 / a
\end{array}\right] .
$$

We obtain

$$
\widetilde{\omega}^{1}=a^{-1} \omega^{1}, \quad \widetilde{\omega}^{2}=a \omega^{2}, \quad \widetilde{\omega}_{1}^{2}=a^{2} \omega_{1}^{2} .
$$

Hence

$$
0=\omega_{1}^{2}-p \omega^{1}=a^{-2} \widetilde{\omega}_{1}^{2}-p a \widetilde{\omega}^{1}=a^{-2}\left(\widetilde{\omega}_{1}^{2}-p a^{3} \widetilde{\omega}^{1}\right) .
$$

The Pfaffian system $\bar{\Omega}^{1}$ is transformed to

$$
\widetilde{\omega}^{2}=0, \quad \widetilde{\omega}_{1}^{2}-p a^{3} \widetilde{\omega}^{1}=0 .
$$

That is, the function $p$ undergoes the transformation $p \mapsto a^{3} p$. Accordingly, we can choose $a$ so that $a p^{3}=1$ and transform $\bar{\Omega}^{1}$ to ${ }^{4}$

$$
\Omega^{2}: \omega^{2}=0, \omega_{1}^{2}-\omega_{1}=0 .
$$

\footnotetext{
${ }^{4}$ We have made a tacit genericity assumption that $p \neq 0$. The case $p=0$ must be considered separately, as in [14]. To simplify the exposition we shall continue to make such genericity assumptions in this paper.
} 
The integral submanifolds of $\Omega^{2}$, with independence form $\omega^{1}$ are the "second order frames" for curves in $\mathbb{A}^{2}$. The subgroup $H_{2} \subset H_{1} \subset H$ that preserves the elements of $\Omega^{2}$ is

$$
\left[\begin{array}{lll}
1 & 0 & 0 \\
0 & 1 & b \\
0 & 0 & 1
\end{array}\right] .
$$

We must now extend $\Omega^{2}$ to a differential ideal by computing the exterior derivative of $\omega_{1}^{2}-\omega^{1}$. From the structure equations we obtain $3 \omega_{1}^{1} \wedge \omega^{1}=0$. As before, there is a function $q$ on $G$ such that the 2 -form equation can be replaced by

$$
\omega_{1}^{1}-q \omega^{1}=0
$$

so that (the reconstituted) $\bar{\Omega}^{2}$ is given by the 1 -form equations

$$
\bar{\Omega}^{2}: \omega^{2}=0, \quad \omega_{1}^{2}-\omega^{1}=0, \quad \omega_{1}^{1}-q \omega^{1}=0
$$

and is no longer a differential ideal.

By performing a $H_{2}$ change of frame the 1-forms in (7) become

$$
\widetilde{\omega}^{2}=\omega^{2}, \quad \widetilde{\omega}_{1}^{2}-\widetilde{\omega}^{1}=\omega_{1}^{2}-\omega^{1}, \quad \widetilde{\omega}_{1}^{1}=\omega_{1}^{1}-b \omega_{1}^{2} .
$$

Thus $\Omega^{2}$ is invariant under a $H_{2}$ change of frame while

$$
0=\omega_{1}^{1}-q \omega^{1}=\widetilde{\omega}_{1}^{1}+(b-q) \widetilde{\omega}^{1} .
$$

We can chose $b=q$ to obtain the 1 -form equation $\widetilde{\omega}_{1}^{1}=0$, and giving rise to the final adapted frame (dropping tildes)

$$
\Omega^{3}: \omega^{2}=0, \omega_{1}^{2}-\omega_{1}=0, \omega_{1}^{1}=0
$$

which "reduces the isotropy group to the identity". Thus, $\Omega^{3}$ is the Pfaffian system $\Omega_{\mathfrak{F}}$ and its integral curves are the Frenet lifts $\mathfrak{F}$ of curves in $\mathbb{A}^{2}$. Computing the exterior derivative of $\omega_{1}^{1}=0$ we obtain the 2 -form equation $\omega_{2}^{1} \wedge \omega^{1}=0$ and hence there is a function $\kappa$ on $G$ such that $\omega_{2}^{1}-\kappa \omega^{1}=0$. This time there is no freedom left in our choice of frame that enables $\kappa$ to be transformed away. The function $\kappa$ here is intrinsic. Hence, in this case, the Cartan prolongation we seek is the Pfaffian system $\Omega_{\mathfrak{F}}$ augmented by the 1 -form equation $\omega_{2}^{1}-\kappa \omega^{1}=0$

$$
\widehat{\Omega}_{\mathfrak{F}}: \omega^{2}=0, \quad \omega_{1}^{2}-\omega^{1}=0, \quad \omega_{1}^{1}=0, \quad \omega_{2}^{1}-\kappa \omega^{1}=0,
$$

on $G \times \mathbb{R}_{\kappa}$. The integral curves of $\widehat{\Omega}_{\mathfrak{F}}$ with independence form $\omega^{1}$ determine the unique equiaffine invariant for plane curves.

Theorem 1. Let $I \subseteq \mathbb{R}$ be an interval and $\gamma_{i}: I \rightarrow \mathbb{A}^{2}$ be two immersed curves in the equi-affine plane, each parametrised by equi-affine arc-length. Then there is an element $g \in G$ such that $\gamma_{2}=g \cdot \gamma_{1}$ if and only if their Cartan lifts $\widehat{\Gamma}_{i}: I \rightarrow G \times \mathbb{R}$ satisfy

$$
\widehat{\Gamma}_{1}^{*} \kappa=\widehat{\Gamma}_{2}^{*} \kappa
$$

identically on $I$.

Proof. Let us firstly recall that $\left(G \times \mathbb{R}, \widehat{\Omega}_{\mathfrak{F}}\right) \rightarrow\left(G, \Omega_{\mathfrak{F}}\right)$ is a Cartan prolongation and $\widehat{\Gamma}_{i}$ is a Cartan lift of $\Gamma_{i}, i=1,2$; see diagram (5). By definition, the $\Gamma_{i}$ are the Frenet lifts of $\gamma_{i}$ 
and $\widehat{\Gamma}_{i}$ are Cartan lifts of $\gamma_{i}$. Finally $\widehat{\Gamma}_{i}$ are integral submanifolds of $\widehat{\Omega}_{\mathfrak{F}}$ on $G \times \mathbb{R}$ and consquently for $i=1,2$ we have

$$
\widehat{\Gamma}_{i}^{*} \omega^{2}=\widehat{\Gamma}_{i}^{*} \omega_{1}^{1}=0, \quad \widehat{\Gamma}_{i}^{*} \omega_{2}^{1}=\left(\widehat{\Gamma}_{i}^{*} \kappa\right)\left(\widehat{\Gamma}_{i}^{*} \omega^{1}\right), \quad \widehat{\Gamma}_{i}^{*} \omega_{1}^{2}=\widehat{\Gamma}_{i}^{*} \omega^{1} .
$$

Since both curves are parametrised by equi-affine arc-length $s$ we have $\widehat{\Gamma}_{1}^{*} \omega^{1}=\widehat{\Gamma}_{2}^{*} \omega^{1}=d s$. From this and from (9) we deduce that

$$
\Gamma_{1}^{*} \Omega_{\mathrm{MC}}=\Gamma_{2}^{*} \Omega_{\mathrm{MC}}
$$

where $\Omega_{\mathrm{MC}}$ is the Maurer-Cartan form on $G$. It follows from the standard theorem about maps into a Lie group [22, Chapter 10, Theorem 18] that there is a fixed element $g \in G$ such that $\gamma_{2}=g \cdot \gamma_{1}$.

Conversely, if $\gamma_{2}=g \cdot \gamma_{1}$ for some $g \in G$, then the Frenet lifts $\Gamma_{i}$ of $\gamma_{i}$ satisfy (10) and are integral submanifolds of $\Omega_{\mathfrak{F}}$. But since $\widehat{\Omega}_{\mathfrak{F}}$ is a Cartan prolongation of $\Omega_{\mathfrak{F}}$, there are Cartan lifts $\widehat{\Gamma}_{i}$ of $\Gamma_{i}$ which are integral submanifolds of $\widehat{\Omega}_{\mathfrak{F}}$. Equation (8) follows from this and equation (10).

Remark 1. This theorem encapsulates the basic idea of this paper and is proposed as a model for the study of curves in any Cartan geometry. The relationship between Theorem 1 and diagram (5) should be clear. The idea now is that by the Goursat normal form $\left(G \times \mathbb{R}, \widehat{\Omega} \frac{1}{\mathfrak{F}}\right)$ is locally diffeomorphic to the jet bundle $\left(J^{4}(\mathbb{R}, \mathbb{R}), \mathcal{C}_{1}^{(4)}\right)$, where $\mathcal{C}_{1}^{(4)}$ is the contact sub-bundle of $T J^{4}(\mathbb{R}, \mathbb{R})$. That is, there is a local diffeomorphism $\phi: G \times \mathbb{R} \rightarrow J^{4}(\mathbb{R}, \mathbb{R})$ such that $\phi_{*} \widehat{\Omega}_{\mathfrak{F}}^{\perp}=\mathcal{C}_{1}^{(4)}$. In fact, Theorem 1 proves that knowing the diffeomorphism $\phi$ explicitly constructs the unique invariant $\kappa$ for plane equi-affine curves explicitly, namely the equi-affine curvature, as well as the equi-affine arc-length. Explaining this is the goal of the next subsection.

\subsection{Goursat normal form}

The Goursat normal form is a local characterisation of the contact distribution on $J^{k}(\mathbb{R}, \mathbb{R})$ for all $k \geq 1$, which we denote $\mathcal{C}_{1}^{(k)}$. The original theorem is not due to Goursat who was its populariser. It appears the theorem is originally due, in some form, to E. von Weber but the statement of it I give below essentially arises from a 1914 work of Cartan. A good reference is [25]. This reference describes an interesting, relevant but largely forgotten work of Vessiot [29]. First we establish some notation and definitions.

\subsubsection{The derived flag}

Suppose $M$ is a smooth manifold and $\mathcal{V} \subset T M$ a smooth sub-bundle of its tangent bundle. The structure tensor is the homomorphism of vector bundles $\delta: \Lambda^{2} \mathcal{V} \rightarrow T M / \mathcal{V}$ defined by

$$
\delta(X, Y)=[X, Y] \bmod \mathcal{V}, \quad \text { for } X, Y \in \Gamma(M, \mathcal{V}) .
$$

If $\delta$ has constant rank, we define the first derived bundle $\mathcal{V}^{(1)}$ as the inverse image of $\delta\left(\Lambda^{2} \mathcal{V}\right)$ under the canonical projection $T M \rightarrow T M / \mathcal{V}$. Informally,

$$
\mathcal{V}^{(1)}=\mathcal{V}+[\mathcal{V}, \mathcal{V}]
$$

The derived bundles $\mathcal{V}^{(i)}$ are defined inductively:

$$
\mathcal{V}^{(i+1)}=\mathcal{V}^{(i)}+\left[\mathcal{V}^{(i)}, \mathcal{V}^{(i)}\right]
$$


assuming that at each iteration it defines a vector bundle, in which case we shall say that $\mathcal{V}$ is regular. For regular $\mathcal{V}$, by dimension reasons, there will be a smallest $k$ for which $\mathcal{V}^{(k+1)}=\mathcal{V}^{(k)}$. This $k$ is called the derived length of $\mathcal{V}$ and the whole sequence of sub-bundles

$$
\mathcal{V} \subset \mathcal{V}^{(1)} \subset \mathcal{V}^{(2)} \subset \cdots \subset \mathcal{V}^{(k)}
$$

the derived flag of $\mathcal{V}$. We shall denote by $\mathcal{V}^{(\infty)}$ the smallest integrable sub-bundle containing $\mathcal{V}$.

\subsubsection{Cauchy bundles}

Let us define

$$
\sigma: \mathcal{V} \rightarrow \operatorname{Hom}(\mathcal{V}, T M / \mathcal{V}) \quad \text { by } \sigma(X)(Y)=\delta(X, Y)
$$

Even if $\mathcal{V}$ is regular, the homomorphism $\sigma$ need not have constant rank. If it does, let us write Char $\mathcal{V}$ for its kernel. The Jacobi identity shows that Char $\mathcal{V}$ is always integrable. It is called the Cauchy bundle or characteristic bundle of $\mathcal{V}$. If $\mathcal{V}$ is regular and each $\mathcal{V}^{(i)}$ has a Cauchy bundle then, we say that $\mathcal{V}$ is totally regular. Then by the derived type of $\mathcal{V}$ we shall mean the list $\left\{\mathcal{V}^{(i)}\right.$, Char $\left.\mathcal{V}^{(i)}\right\}$ of subundles.

Theorem 2 (Goursat normal form). Let $\mathcal{V} \subset T M$ be a smooth, totally regular, rank 2 sub-bundle over smooth manifold $M$ such that

a) $\mathcal{V}^{(\infty)}=T M$;

b) $\operatorname{dim} \mathcal{V}^{(i+1)}=\operatorname{dim} \mathcal{V}^{(i)}+1$, while $\mathcal{V}^{(i)} \neq T M$.

Then there is a generic subset $\hat{M} \subseteq M$ such that in a neigbourhood of each point of $\hat{M}$ there are local coordinates $x, z_{0}, z_{1}, z_{2}, \ldots z_{k}$ such that $\mathcal{V}$ has local expression

$$
\left\{\partial_{x}+\sum_{j=1}^{k} z_{j} \partial_{z_{j-1}}, \partial_{z_{k}}\right\},
$$

where $k=\operatorname{dim} M-2$. That is, $\mathcal{V}$ is locally equivalent to $\mathcal{C}_{1}^{(k)}$ on $\hat{M}$.

A proof can be found in [25, pp. 157-159]. The proof of a much more general result in which the Goursat normal form is a special case is given in [28]. The significance of the latter is that an procedure is provided for constructing the local contact coordinates $x, z_{0}, \ldots, z_{k}$. This is procedure Contact $B$ on page 287 of [28] with $\rho_{k}=1$ and $\rho_{1}=\rho_{2}=\cdots=\rho_{k-1}=0$; the $\rho_{i}$ are defined in Section 3. In this special case we have the following.

\section{Procedure Contact for the Goursat normal form}

INPUT : Sub-bundle $\mathcal{V} \subset T M$ of derived length $k$ which satisfies the hypotheses of Theorem 2.

a) Fix any first integral of Char $\mathcal{V}^{(k-1)}$, denoted $x$, and any section $\boldsymbol{Z}$ of $\mathcal{V}$ such that $\boldsymbol{Z} x=1$.

b) Define a distribution $\Pi^{k}$ as follows:

$$
\Pi^{l+1}=\left[Z, \Pi^{l}\right], \quad \Pi^{1}=\text { Char } \mathcal{V}^{(1)}, \quad 1 \leq l \leq k-1 .
$$

c) Let $z_{0}$ be any invariant of $\Pi^{k}$ such that $d x \wedge d z_{0} \neq 0$.

d) Define functions $z_{1}, z_{2}, \ldots, z_{k}$ by $z_{j}=Z_{z_{j-1}}, j=1, \ldots, k$.

OUTPUT : Functions $x, z_{0}, z_{1}, \ldots, z_{k}$ are contact coordinates for $\mathcal{V}$.

The proof of correctness of this procedure is given in [28]. 


\subsection{Equi-affine invariants \& the Goursat normal form}

We use this procedure to construct the various invariant objects for this geometry. In fact we will construct the Frenet frames by constructing all the integral submanifolds of $\widehat{\Omega}_{\mathfrak{F}}$. So we set $\mathcal{V}:=\widehat{\Omega}_{\mathfrak{F}}^{\perp}$ :

$$
\mathcal{V}=\left\{\partial_{\omega^{1}}+\partial_{\omega_{1}^{2}}+\kappa \partial_{\omega_{2}^{1}}, \partial_{\kappa}\right\}
$$

Note that we have adopted the usual convention of denoting the frame dual to

$$
\omega^{1}, \quad \omega^{2}, \quad \omega_{1}^{1}, \quad \omega_{2}^{1}, \quad \omega_{1}^{2}
$$

by

$$
\partial_{\omega^{1}}, \quad \partial_{\omega^{2}}, \quad \partial_{\omega_{1}^{1}}, \quad \partial_{\omega_{2}^{1}}, \partial_{\omega_{1}^{2}}
$$

In local coordinates we have

$$
\mathcal{V}=\left\{X:=a \partial_{x}+c \partial_{y}+b \partial_{a}+\kappa a \partial_{b}+\frac{1+b c}{a} \partial_{c}, \partial_{\kappa}\right\}
$$

Calculation verifies that the hypotheses of Theorem 2 are met and that the derived length of $\mathcal{V}$ is $k=4$. Then step a) of Contact requires that we construct at least one invariant of

$$
\text { Char } \mathcal{V}^{(3)}=\left\{\partial_{b}, \quad \partial_{\kappa}, \quad a \partial_{a}+c \partial_{c}\right\}
$$

which has invariants $x, y, a / c$. Any one of these can be taken as the "independent variable". Since $x, y$ are local coordinates on $G / S L(2, \mathbb{R})$, we shall choose $x$ for this purpose. It then follows that we may take $\boldsymbol{Z}$ to be

$$
\boldsymbol{Z}=\frac{1}{a} X=\partial_{x}+\frac{c}{a} \partial_{y}+\frac{b}{a} \partial_{a}+\kappa \partial_{b}+\frac{1+b c}{a^{2}} \partial_{c} .
$$

Step b) requires the construction of $\Pi^{4}$. We get

$$
\begin{aligned}
& \Pi^{1}=\left\{\partial_{\kappa}\right\}, \quad \Pi^{2}=\left\{\partial_{\kappa}, \partial_{b}\right\}, \quad \Pi^{3}=\left\{\partial_{\kappa}, \partial_{b}, \frac{1}{a} \partial_{a}+\frac{c}{a^{2}} \partial_{c}\right\}, \\
& \Pi^{4}=\left\{\partial_{\kappa}, \partial_{b}, \frac{1}{a} \partial_{a}+\frac{c}{a^{2}} \partial_{c}, \partial_{c}\right\} .
\end{aligned}
$$

The invariants of $\Pi^{4}$ are in fact $x, y$ and hence by step c), we set $z_{0}=y$ and construct $z_{j}=\boldsymbol{Z} z_{j-1}$, $1 \leq j \leq 4$. We get

$$
z_{1}=\frac{c}{a}, \quad z_{2}=\frac{1}{a^{3}}, \quad z_{3}=-\frac{3 b}{a^{5}}, \quad z_{4}=3 \frac{5 b^{2}-a^{2} \kappa}{a^{7}}
$$

obtaining the equivalence $\phi: U \subset G \times \mathbb{R} \rightarrow J^{4}(\mathbb{R}, \mathbb{R})$ defined by

$$
\phi(x, y, a, b, c, \kappa)=\left(x, y, c a^{-1}, a^{-3},-3 b a^{-5}, 3\left(5 b^{2}-a^{2} \kappa\right) a^{-7}\right)
$$

between $\mathcal{V}$ and the contact distribution on $J^{4}(\mathbb{R}, \mathbb{R})$. The inverse of $\phi$ is

$$
\begin{aligned}
\phi^{-1}\left(x, z_{0}, z_{1}, z_{2}, z_{3}, z_{4}\right) & =\left(x, z_{0}, z_{2}^{-1 / 3},-3^{-1} z_{3} z_{2}^{-5 / 3}, z_{1} z_{2}^{-1 / 3}, 9^{-1}\left(5 z_{3}^{2}-3 z_{2} z_{4}\right) z_{2}^{-8 / 3}\right) \\
& =(x, y, a, b, c, \kappa) .
\end{aligned}
$$


Hence if we express the curve in $\mathbb{A}^{2}$ as a graph $(x, f(x))$ then we deduce from $\phi^{-1}$ that the equi-affine curvature is the well known expression

$$
\kappa:=\frac{5 f^{\prime \prime \prime}(x)^{2}-3 f^{\prime \prime}(x) f^{\prime \prime \prime \prime}(x)}{9 f^{\prime \prime}(x)^{8 / 3}}=\frac{1}{2}\left(f^{\prime \prime}(x)^{-2 / 3}\right)^{\prime \prime} .
$$

We also obtain the unique $G$-invariant 1 -form, the equi-affine arc length by pulling back $\omega^{1}$ by $\phi^{-1}$,

$$
\left(\phi^{-1}\right)^{*} \omega^{1}=f^{\prime \prime}(x)^{1 / 3} d x
$$

and the Frenet frame

$$
\mathfrak{F}=\left(\begin{array}{ccc}
1 & 0 & 0 \\
x & f^{\prime \prime}(x)^{-1 / 3} & -3^{-1} f^{\prime \prime \prime}(x) f^{\prime \prime}(x)^{-5 / 3} \\
f(x) & f^{\prime}(x) f^{\prime \prime}(x)^{-1 / 3} & \left(f^{\prime \prime}(x)^{2}-3^{-1} f^{\prime \prime \prime}(x) f^{\prime}(x)\right) f^{\prime \prime}(x)^{-5 / 3}
\end{array}\right)
$$

of the curve $(x, f(x))$ by pulling back an arbitrary element $g \in G$ by $\phi^{-1}$. Of course, we can express everything in terms an arbitrary immersion $(x(t), y(t))$ into $\mathbb{A}^{2}$, rather than as a graph.

Remark 2. Note that the procedure we have just described for the invariant data of curves in $\mathbb{A}^{2}$ is not algorithmic; we had to solve differential equations to obtain the equivalence $\phi$. In practice, however, we find that when the contact system is that of $J^{k}\left(\mathbb{R}, \mathbb{R}^{q}\right)$ where $q>1$, this integration can often be avoided. We will illustrate this for curves in $\mathbb{A}^{3}$ and prove, in Section 5 , that it holds for curves in any Riemannian manifold of dimension greater than 2 .

\section{On the generalised Goursat normal form}

To carry out the programme of the previous section for curves immersed in manifolds of dimension greater than two we must be able to characterise the contact distributions on jet spaces $J^{k}\left(\mathbb{R}, \mathbb{R}^{q}\right)$, for all $k, q \geq 1$; the case $q=1$ being the Goursat normal form. In principle this generalisation should include partial prolongations of the contact distribution on $J^{1}\left(\mathbb{R}, \mathbb{R}^{q}\right)$ and such a characterisation exists - the generalised Goursat normal form [27, 28]. However, so far the full scope of this characterisation has not been required. It turns out that only total prolongations of the first order jet space are sufficient. Accordingly, we will only briefly review those parts of $[27,28]$ that are needed for the results to be described in this paper.

\subsection{The singular variety}

For each $x \in M$, let

$$
\mathcal{S}_{x}=\left\{v \in \mathcal{V}_{x} \backslash 0 \mid \sigma(v) \text { has less than generic rank }\right\} .
$$

Then $\mathcal{S}_{x}$ is the zero set of homogeneous polynomials and so defines a subvariety of the projectivisation $\mathbb{P} \mathcal{V}_{x}$ of $\mathcal{V}_{x}$. We shall denote by $\operatorname{Sing}(\mathcal{V})$ the fibre bundle over $M$ with fibre over $x \in M$ equal to $\mathcal{S}_{x}$ and we refer to it as the singular variety of $\mathcal{V}$. For $X \in \mathcal{V}$ the matrix of the homomorphism $\sigma(X)$ will be called the polar matrix of $[X] \in \mathbb{P V}$. There is a map $\operatorname{deg}_{\mathcal{V}}: \mathbb{P} \mathcal{V} \rightarrow \mathbb{N}$ well defined by

$$
\operatorname{deg}_{\mathcal{V}}([X])=\operatorname{rank} \sigma(X) \quad \text { for }[X] \in \mathbb{P} \mathcal{V} .
$$

We shall call $\operatorname{deg}_{\mathcal{V}}([X])$ the degree of $[X]$. The singular variety $\operatorname{Sing}(\mathcal{V})$ is a diffeomorphism invariant in the sense that if $\mathcal{V}_{1}, \mathcal{V}_{2}$ are sub-bundles over $M_{1}, M_{2}$, respectively and there is 
a diffeomorphism $\phi: M_{1} \rightarrow M_{2}$ that identifies them, then $\operatorname{Sing}\left(\mathcal{V}_{2}\right)$ and $\operatorname{Sing}\left(\phi_{*} \mathcal{V}_{1}\right)$ are equivalent as projective subvarieties of $\mathbb{P} \mathcal{V}_{2}$. That is, for each $x \in M_{1}$, there is an element of the projective linear group $P G L\left(\mathcal{V}_{\left.2\right|_{\phi(x)}}, \mathbb{R}\right)$ that identifies $\operatorname{Sing}\left(\mathcal{V}_{2}\right)(\phi(x))$ and $\operatorname{Sing}\left(\phi_{*} \mathcal{V}_{1}\right)(\phi(x))$.

We hasten to point out that the computation of the singular variety for any given subbundle $\mathcal{V} \subset T M$ is algorithmic. One computes the determinantal variety of the polar matrix for generic $[X]$.

\subsubsection{The singular variety in positive degree}

If $X \in$ Char $\mathcal{V}$ then $\operatorname{deg}_{\mathcal{V}}([X])=0$. It is convenient to eliminate lines of degree zero and for this reason we pass to the quotient $\widehat{\mathcal{V}}:=\mathcal{V} /$ Char $\mathcal{V}$. We have structure tensor $\widehat{\delta}: \Lambda^{2} \widehat{\mathcal{V}} \rightarrow \widehat{T M} / \widehat{\mathcal{V}}$ well defined by

$$
\widehat{\delta}(\widehat{X}, \widehat{Y})=\pi([X, Y]) \bmod \widehat{\mathcal{V}}
$$

where $\widehat{T M}=T M /$ Char $\mathcal{V}$ and

$$
\pi: T M \rightarrow \widehat{T M}
$$

is the canonical projection. The notion of degree descends to this quotient giving a map

$$
\operatorname{deg}_{\widehat{\mathcal{V}}}: \mathbb{P} \widehat{\mathcal{V}} \rightarrow \mathbb{N}
$$

well defined by

$$
\operatorname{deg}_{\widehat{\mathcal{V}}}([\widehat{X}])=\operatorname{rank} \widehat{\sigma}(\widehat{X}) \quad \text { for }[\widehat{X}] \in \mathbb{P} \widehat{\mathcal{V}},
$$

where $\widehat{\sigma}(\widehat{X})(\widehat{Y})=\widehat{\delta}(\widehat{X}, \widehat{Y})$ for $\widehat{Y} \in \widehat{\mathcal{V}}$. Note that all definitions go over mutatis mutandis when the structure tensor $\delta$ is replaced by $\widehat{\delta}$. In particular, we have notions of polar matrix and singular variety, as before. However, if the singular variety of $\widehat{\mathcal{V}}$ is not empty, then each point of $\mathbb{P} \widehat{\mathcal{V}}$ has degree one or more.

\subsubsection{The resolvent bundle}

Suppose $\mathcal{V} \subset T M$ is totally regular of rank $c+q+1, q \geq 2, c \geq 0, \operatorname{dim} M=c+2 q+1$. Suppose further that $\mathcal{V}$ satisfies

a) $\operatorname{dim}$ Char $\mathcal{V}=c, \mathcal{V}^{(1)}=T M$;

b) $\widehat{\Sigma}_{\mid x}:=\operatorname{Sing}(\widehat{\mathcal{V}})_{\mid x}=\mathbb{P} \widehat{\mathcal{B}}_{\mid x} \approx \mathbb{R P}^{q-1}$, for each $x \in M$ and some rank $q$ sub-bundle $\widehat{\mathcal{B}} \subset \widehat{\mathcal{V}}$. Then we call $(\mathcal{V}, \mathbb{P} \widehat{\mathcal{B}})($ or $(\mathcal{V}, \widehat{\Sigma}))$ a Weber structure of rank $q$ on $M$.

Given a Weber structure $(\mathcal{V}, \mathbb{P} \widehat{\mathcal{B}})$, let $\mathcal{R}(\mathcal{V}) \subset \mathcal{V}$, denote the largest sub-bundle such that

$$
\pi(\mathcal{R}(\mathcal{V}))=\widehat{\mathcal{B}}
$$

We call the rank $q+c$ bundle $\mathcal{R}(\mathcal{V})$ defined by (11) the resolvent bundle associated to the Weber structure $(\mathcal{V}, \widehat{\Sigma})$. The bundle $\widehat{\mathcal{B}}$ determined by the singular variety of $\widehat{\mathcal{V}}$ will be called the singular sub-bundle of the Weber structure. A Weber structure will be said to be integrable if its resolvent bundle is integrable.

We will see that the resolvent bundle is the key to the construction of an identification of a given differential system with a contact system, if such an identification exists; and hence the name. 
An integrable Weber structure descends to the quotient of $M$ by the leaves of Char $\mathcal{V}$ to be the contact bundle on $J^{1}\left(\mathbb{R}, \mathbb{R}^{q}\right)$. Thus, the resolvent bundle and its concomitant Weber structure is a constructive characterisation of the contact bundle on the 1-jets $J^{1}\left(\mathbb{R}, \mathbb{R}^{q}\right)$. The term 'Weber structure' honours Eduard von Weber (1870-1934) who was the first to publish a proof of the Goursat normal form. For completeness we record the following properties of the resolvent bundle of a Weber structure.

Proposition 1 ([27]). Let $(\mathcal{V}, \widehat{\Sigma})$ be a Weber structure on $M$ and $\widehat{\mathcal{B}}$ its singular sub-bundle. If $q \geq 3$, then the following are equivalent

a) its resolvent bundle $\mathcal{R}(\mathcal{V}) \subset \mathcal{V}$ is integrable;

b) each point of $\widehat{\Sigma}=\operatorname{Sing}(\widehat{\mathcal{V}})$ has degree one;

c) the structure tensor $\widehat{\delta}$ of $\widehat{\mathcal{V}}$ vanishes on $\widehat{\mathcal{B}}: \widehat{\delta}(\widehat{\mathcal{B}}, \widehat{\mathcal{B}})=0$.

Proposition 2 ([27]). Let $(\mathcal{V}, \widehat{\Sigma})$ be an integrable Weber structure on $M$. Then its resolvent bundle $\mathcal{R}(\mathcal{V})$ is the unique, maximal, integrable sub-bundle of $\mathcal{V}$.

Checking the integrability of the resolvent bundle is algorithmic. One computes the singular variety $\operatorname{Sing}(\widehat{\mathcal{V}})=\mathbb{P} \widehat{\mathcal{B}}$. In turn, the singular bundle $\widehat{\mathcal{B}}$ algorithmically determines $\mathcal{R}(\mathcal{V})$.

Example 1. The canonical model of an integrable Weber structure is the contact distribution on $J^{1}\left(\mathbb{R}, \mathbb{R}^{q}\right)$ for $q>1$, extended by Cauchy characteristics

$$
\mathcal{V}=\left\{\partial_{x}+\sum_{i=1}^{q} p_{i} \partial_{u_{i}}, \partial_{p_{1}}, \ldots, \partial_{p_{q}}, \partial_{z_{1}}, \partial_{z_{2}}, \ldots, \partial_{z_{c}}\right\} .
$$

The quotient $\widehat{\mathcal{V}}=\mathcal{V} /$ Char $\mathcal{V}$ has singular sub-bundle

$$
\widehat{\mathcal{B}}=\left\{\left[\partial_{p_{1}}\right], \ldots,\left[\partial_{p_{q}}\right]\right\}
$$

and the resolvent bundle of integrable Weber structure $(\mathcal{V}, \mathbb{P} \widehat{\mathcal{B}})$ is

$$
\mathcal{R}(\mathcal{V})=\left\{\partial_{p_{1}}, \ldots, \partial_{p_{q}}, \partial_{z_{1}}, \partial_{z_{2}}, \ldots, \partial_{z_{c}}\right\}
$$

The invariants of the resolvent bundle are spanned by $\left\{x, u_{1}, \ldots, u_{q}\right\}$. So the resolvent bundle provides a geometric characterisation of the "independent variable" $x$ and the "dependent variables" $u_{i}$, after which differentiation by a canonically defined total derivative operator leads to higher order jet coordinates. See Sections 3.2 and 4 of this paper for further details. See [27, 28] for the general theory with proofs and further examples.

\subsection{The uniform generalised Goursat normal form}

We are now able to give a characterisation of the contact distribution on $J^{k}\left(\mathbb{R}, \mathbb{R}^{q}\right), \mathcal{C}_{q}^{(k)}$ for any $k, q \geq 1$, generalising the Goursat normal form to the uniform case.

Theorem 3 (generalised Goursat normal form - uniform case, [27, 28]). Let $\mathcal{V} \subset T M$ be a smooth, totally regular, sub-bundle of rank $q+1$ and derived length $k$, some $k, q>0$, over smooth manifold $M$ such that

a) $\mathcal{V}^{(\infty)}=T M$;

b) $\operatorname{dim} \mathcal{V}^{(i+1)}=\operatorname{dim} \mathcal{V}^{(i)}+q$, while $\mathcal{V}^{(i)} \neq T M$;

c) Char $\mathcal{V}^{(i)} \subset \mathcal{V}^{(i-1)}, 1 \leq i \leq k-1$; $\operatorname{dim} \operatorname{Char} \mathcal{V}^{(j)}=j q, 0 \leq j \leq k-1$;

d) If $q>1$ then $\mathcal{V}^{(k-1)}$ admits an integrable Weber structure.

Then there is a generic subset $\hat{M} \subseteq M$ such that around each point of $\hat{M}$ the distribution $\mathcal{V}$ is locally equivalent to $\mathcal{C}_{q}^{(k)}$. 
If $q=1$ then $\mathrm{c}$ ) follows from b) and the Weber structure is not defined in which case Theorem 3 reduces to the Goursat normal form, Theorem 2. We call any sub-bundle that satisfies the hypotheses of Theorem 3 a uniform Goursat bundle in which case the Theorem asserts that generically every uniform Goursat bundle is locally equivalent to the canonical one. A proof of Theorem 3 can be found in [27] as a special case of that paper's Theorem 4.1. However, the latter covers a very much larger class of sub-bundles than uniform Goursat bundles and there is therefore a much simpler proof in this uniform case. However, for the purposes of this paper an important thing is the procedure for constructing contact coordinates in the uniform case which is a special case of procedure Contact $A$ on page 286 of $[28]^{5}$ with $\rho_{1}=\rho_{2}=\cdots=\rho_{k=1}=0$, $\rho_{k}=q>1$. Note that the collection of non-negative integers

$$
\sigma=\left\langle\rho_{1}, \rho_{2}, \ldots, \rho_{k}\right\rangle
$$

shall be called the signature of $\mathcal{V}$ and is a complete local invariant of Goursat bundles. In the interests of completeness we mention that the non-negative integers $\rho_{i}$ are defined by

$$
\begin{aligned}
& \rho_{j}=\operatorname{dim} \operatorname{Char} \mathcal{V}^{(j)}-\operatorname{dim} \operatorname{Char} \mathcal{V}_{j-1}^{(j)}, \quad 1 \leq j \leq k-1, \\
& \rho_{k}=\operatorname{dim} \mathcal{V}^{(k)}-\operatorname{dim} \mathcal{V}^{(k-1)},
\end{aligned}
$$

where Char $\mathcal{V}_{j-1}^{(j)}=$ Char $\mathcal{V}^{(j)} \cap \mathcal{V}^{(j-1)}$. It is proved in [27, 28] that a sub-bundle $\mathcal{V}$ on manifold $M$ is locally diffeomorphic to a partial prolongation of the contact system $\mathcal{C}_{q}^{(1)}$ on $J^{1}\left(\mathbb{R}, \mathbb{R}^{q}\right)$ with $\rho_{j}$ variables of order $j$, if and only if $(M, \mathcal{V})$ is a Goursat bundle of signature $\sigma$. In this paper, we need only consider total prolongations in which the only nonzero element of the signature is $\rho_{k}$, where $k$ is the derived length of $\mathcal{V}$.

\section{Procedure Contact for uniform generalised Goursat bundles, $q>1$}

INPUT : Uniform Goursat bundle $\mathcal{V} \subset T M$ of derived length $k$ with $q>1$.

a) Construct the (integrable) resolvent bundle $\mathcal{R}\left(\mathcal{V}^{(k-1)}\right)$ and all its $q+1$ first integrals.

b) Fix any one of the first integrals from step a) denoted $x$, and any section $\boldsymbol{Z}$ of $\mathcal{V}$ such that $\boldsymbol{Z} x=1$.

c) Denote the remaining $q$ first integrals of $\mathcal{R}\left(\mathcal{V}^{(k-1)}\right)$ by $z_{0}^{j}, j=1,2, \ldots, q$.

d) Define functions $z_{1}^{j}, z_{2}^{j}, \ldots, z_{k}^{j}$ by $z_{m}^{j}=\boldsymbol{Z} z_{m-1}^{j}, 1 \leq m \leq k, 1 \leq j \leq q$.

OUTPUT : Functions $x, z_{0}^{j}, z_{1}^{j}, \ldots, z_{k}^{j}, 1 \leq j \leq q$ are contact coordinates for $\mathcal{V}$.

Remark 3. Even though an integration problem is presented for solution in step a) above, in fact, in every example of curves in a Cartan geometry that I've seen no integration is required because the resolvent bundle turns out to be the vertical bundle for the fibration $\pi \circ \widehat{\pi}$ : $E \rightarrow G / H$. So a complete set of invariants of $\mathcal{R}\left(\mathcal{V}^{(k-1)}\right)$ can be taken to be the components of any coordinate system on $G / H$. In Section 5 we will prove this for curves in any Riemannian manifold of dimension at least 3 .

\section{Space curves up to equi-affine transformations}

As an illustration of the generalised Goursat normal form and its relation to the geometry of curves we consider immersed curves in $\mathbb{R}^{3}$ up to the standard action of $G:=S L(3, \mathbb{R}) \ltimes \mathbb{R}^{3}$. The goal is to use repère mobile and Theorem 3 to construct all the invariant data for this situation.

\footnotetext{
${ }^{5}$ Unfortunately in reference [28] procedure Contact was called an algorithm. Manifestly, it does not qualify as an algorithm because in a certain step an integration is required.
} 
We discuss this example principally for illustration since we permit ourselves to begin with the explicit transitive action. However, our goal in this paper is to drop any reliance on an a priori knowledge of a group action. To that end a contention of this paper is that explicit invariant curve data can be obtained without integration or explicit knowledge of the group action in a significant special case, namely Riemannian geometry. This will be established in Section 5 .

A straightforward extension of the $n=2$ case covered in Section 2.1 to the $n=3$ case leads to the matrix group with elements

$$
g=\left(\begin{array}{cccc}
1 & 0 & 0 & 0 \\
x & a_{1} & a_{2} & a_{3} \\
y & a_{4} & a_{5} & a_{6} \\
z & a_{7} & a_{8} & a_{9}
\end{array}\right),
$$

where $\operatorname{det} g=1$. We parametrise an open subset of the group $G$ by solving $\operatorname{det} g=1$ for $a_{9}$. From equations (1), (2) or otherwise we deduce the left-invariant Maurer-Cartan form

$$
\omega=\left(\begin{array}{cccc}
0 & 0 & 0 & 0 \\
\omega^{1} & \omega_{1}^{1} & \omega_{2}^{1} & \omega_{3}^{1} \\
\omega^{2} & \omega_{1}^{2} & \omega_{2}^{2} & \omega_{3}^{2} \\
\omega^{3} & \omega_{1}^{3} & \omega_{2}^{3} & \omega_{3}^{3}
\end{array}\right),
$$

where $\omega_{1}^{1}+\omega_{2}^{2}+\omega_{3}^{3}=0$. For a coframe on $G$ we take the ordered list

$$
\left[\omega^{1}, \omega^{2}, \omega^{3}, \omega_{1}^{1}, \omega_{1}^{2}, \omega_{1}^{3}, \omega_{2}^{1}, \omega_{2}^{2}, \omega_{2}^{3}, \omega_{3}^{1}, \omega_{3}^{2}\right]
$$

whose dual frame we label $\mathfrak{V}=\left[v_{1}, v_{2}, \ldots, v_{11}\right]$. The Lie algebra multiplication table for $\mathfrak{V}$ is

\begin{tabular}{c|ccccccccccc} 
& $v_{1}$ & $v_{2}$ & $v_{3}$ & $v_{4}$ & $v_{5}$ & $v_{6}$ & $v_{7}$ & $v_{8}$ & $v_{9}$ & $v_{10}$ & $v_{11}$ \\
\hline$v_{1}$ & 0 & 0 & 0 & $-v_{1}$ & $-v_{2}$ & $-v_{3}$ & 0 & 0 & 0 & 0 & 0 \\
$v_{2}$ & 0 & 0 & 0 & 0 & 0 & 0 & $-v_{1}$ & $-v_{2}$ & $-v_{3}$ & 0 & 0 \\
$v_{3}$ & 0 & 0 & 0 & $v_{3}$ & 0 & 0 & 0 & $v_{3}$ & 0 & $-v_{1}$ & $-v_{2}$ \\
$v_{4}$ & $v_{1}$ & 0 & $-v_{3}$ & 0 & $-v_{5}$ & $-2 v_{6}$ & $v_{7}$ & 0 & $-v_{9}$ & $2 v_{10}$ & $v_{11}$ \\
$v_{5}$ & $v_{2}$ & 0 & 0 & $v_{5}$ & 0 & 0 & $v_{8}-v_{4}$ & $-v_{5}$ & $-v_{6}$ & $v_{11}$ & 0 \\
$v_{6}$ & $v_{3}$ & 0 & 0 & $2 v_{6}$ & 0 & 0 & $v_{9}$ & $v_{6}$ & 0 & $-v_{4}$ & $-v_{5}$ \\
$v_{7}$ & 0 & $v_{1}$ & 0 & $-v_{7}$ & $v_{4}-v_{8}$ & $-v_{9}$ & 0 & $v_{7}$ & 0 & 0 & $v_{10}$ \\
$v_{8}$ & 0 & $v_{2}$ & $-v_{3}$ & 0 & $v_{5}$ & $-v_{6}$ & $-v_{7}$ & 0 & $-2 v_{9}$ & $v_{10}$ & $2 v_{11}$ \\
$v_{9}$ & 0 & $v_{3}$ & 0 & $v_{9}$ & $v_{6}$ & 0 & 0 & $2 v_{9}$ & 0 & $-v_{7}$ & $-v_{8}$ \\
$v_{10}$ & 0 & 0 & $v_{1}$ & $-2 v_{10}$ & $-v_{11}$ & $v_{4}$ & 0 & $-v_{10}$ & $v_{7}$ & 0 & 0 \\
$v_{11}$ & 0 & 0 & $v_{2}$ & $-v_{11}$ & 0 & $v_{5}$ & $-v_{10}$ & $-2 v_{11}$ & $v_{8}$ & 0 & 0
\end{tabular}

By a procedure similar to the one carried out in the $n=2$ case we arrive at the Pfaffian system $\Omega_{\mathfrak{F}}$ (see [8] for details) whose integral curves are Frenet lifts of curves in $G / S L(3, \mathbb{R})$

$$
\begin{aligned}
\Omega_{\mathfrak{F}}: & \omega^{2}=0, \quad \omega^{3}=0, \quad \omega_{1}^{3}=0, \quad \omega_{1}^{2}-\omega^{1}=0, \quad \omega_{2}^{3}-\omega^{1}=0, \quad \omega_{1}^{1}=0, \\
\omega_{2}^{2} & =0, \quad \omega_{3}^{2}-3 \omega_{2}^{1}=0 .
\end{aligned}
$$

I want to show that a Cartan prolongation of $\Omega_{\mathfrak{F}}$ is a contact system. We calculate that

$$
\Omega_{\mathfrak{F}}^{\perp}=\left\{v_{1}+v_{5}+v_{9}, v_{7}+3 v_{11}, v_{10}\right\} .
$$

The Cartan prolongation we shall consider is obtained from this:

$$
\widehat{\Omega}_{\mathfrak{F}}^{\perp}:\left\{v_{1}+v_{5}+v_{9}+\kappa_{1}\left(v_{7}+3 v_{11}\right)+\kappa_{2} v_{10}, \partial_{\kappa_{1}}, \partial_{\kappa_{2}}\right\}
$$

defined over $G \times \mathbb{R}^{2}$. To apply the generalised Goursat normal form, Theorem 3, we work with this dual bundle $\mathcal{V}:=\widehat{\Omega} \frac{\perp}{\mathfrak{F}}$ and calculate 


\begin{tabular}{|c|c|c|}
\hline$i$ & $\mathcal{V}^{(i)}$ & Char $\mathcal{V}^{(i)}$ \\
\hline 0 & $\left\{v_{1}+v_{5}+v_{9}+\kappa_{1}\left(v_{7}+3 v_{11}\right)+\kappa_{2} v_{10}, \partial_{\kappa_{1}}, \partial_{\kappa_{2}}\right\}$ & $\{0\}$ \\
\hline 1 & $\mathcal{V} \oplus\left\{v_{7}+3 v_{11}, v_{10}\right\}$ & $\left\{\partial_{\kappa_{1}}, \partial_{\kappa_{2}}\right\}$ \\
\hline 2 & $\mathcal{V}^{(1)} \oplus\left\{v_{4}+2 v_{8}, v_{7}-v_{11}\right\}$ & Char $\mathcal{V}^{(1)} \oplus\left\{v_{7}+3 v_{11}, v_{10}\right\}$ \\
\hline 3 & $\mathcal{V}^{(2)} \oplus\left\{v_{4}-2 v_{8}, v_{1}+v_{5}-5 v_{9}\right\}$ & Char $\mathcal{V}^{(2)} \oplus\left\{v_{4}+2 v_{8}, v_{7}-v_{11}\right\}$ \\
\hline 4 & $\mathcal{V}^{(3)} \oplus\left\{v_{6}, v_{1}-3 v_{5}\right\}$ & Char $\mathcal{V}^{(3)} \oplus\left\{v_{4}-2 v_{8}, v_{9}\right\}$ \\
\hline 5 & $T\left(G \times \mathbb{R}^{2}\right)$ & $T\left(G \times \mathbb{R}^{2}\right)$ \\
\hline
\end{tabular}

So hypotheses a), b) and c) of Theorem 3 are satisfied with $q=2$ and derived length $k=5$. Since $q>1$, it remains to check the singular variety of the quotient $\mathcal{V}^{(4)} / \operatorname{Char} \mathcal{V}^{(4)}$. From the table we see that

$$
\text { Char } \mathcal{V}^{(4)}=\left\{v_{4}, v_{7}, v_{8}, v_{9}, v_{10}, v_{11}, \partial_{\kappa_{1}}, \partial_{\kappa_{2}}\right\}
$$

and

$$
\mathcal{V}^{(4)}=\left\{v_{1}, v_{4}, v_{5}, v_{6}, v_{7}, v_{8}, v_{9}, v_{10}, v_{11}, \partial_{\kappa_{1}}, \partial_{\kappa_{2}}\right\}
$$

and hence

$$
\widehat{\mathcal{V}}^{(4)}:=\mathcal{V}^{(4)} / \operatorname{Char} \mathcal{V}^{(4)}=\left\{\left[v_{1}\right],\left[v_{5}\right],\left[v_{6}\right]\right\} .
$$

We obtain that $\operatorname{Im} \hat{\delta}_{4}=\left\{\left[v_{2}\right],\left[v_{3}\right]\right\}$ and the polar matrix of the point $\left\langle a_{1}\left[v_{1}\right]+a_{2}\left[v_{5}\right]+a_{3}\left[v_{6}\right]\right\rangle \in$ $\mathbb{P} \widehat{\mathcal{V}}^{(4)}$ is

$$
\left(\begin{array}{ccc}
-a_{2} & a_{1} & 0 \\
-a_{3} & 0 & a_{1}
\end{array}\right)
$$

which has unit rank if and only if $a_{1}=0$. Hence the singular variety of $\widehat{\mathcal{V}}^{(4)}$ is $\mathbb{R} \mathbb{P}^{1}$ with singular bundle $\widehat{\mathcal{B}}=\left\{\left[v_{5}\right],\left[v_{6}\right]\right\}$. Consequently, the resolvent bundle in this case is

$$
\mathcal{R}\left(\mathcal{V}^{(4)}\right)=\left\{v_{4}, v_{5}, v_{6}, v_{7}, v_{8}, v_{9}, v_{10}, v_{11}\right\} \oplus\left\{\partial_{\kappa_{1}}, \partial_{\kappa_{2}}\right\}=\mathfrak{s l}(3, \mathbb{R}) \oplus \mathbb{R}^{2}
$$

The resolvent bundle is integrable, showing that $\mathcal{V}^{(4)}$ admits an integrable Weber structure, fulfilling hypothesis d) of Theorem 3. We can therefore conclude that $\widehat{\Omega}_{\mathfrak{F}}^{\perp}$ is locally equivalent to the contact distribution $\mathcal{C}_{2}^{(5)}$ on jet space $J^{5}\left(\mathbb{R}, \mathbb{R}^{2}\right)$.

For future reference, we note that we often abuse the term "derived type" by referring to the list of lists

$$
\left[\left[\operatorname{dim} \mathcal{V}^{(j)}, \operatorname{dim} \operatorname{Char} \mathcal{V}^{(j)}\right]\right]_{j=0}^{k}
$$

as the derived type of $\mathcal{V}$, where $k$ is its the derived length. Thus for the example just treated we may say that its derived type is

$$
[[3,0],[5,2],[7,4],[9,6],[11,8],[13,13]]
$$

since it is really the dimensions of these bundles that settles the recognition problem.

\subsection{Differential invariants via Contact}

We now apply procedure Contact to construct the two equi-affine invariants for space curves. In the previous subsection we demonstrated that $\widehat{\Omega}_{\mathfrak{F}}^{\perp}$ is a uniform Goursat bundle. The first step in procedure Contact requires the $q+1=2+1=3$ invariants of the (integrable) resolvent 
bundle, $\mathcal{R}\left(\mathcal{V}^{(4)}\right)$. The vector fields spanning this bundle are all vertical for the projection $G \times \mathbb{R}^{3} \rightarrow \mathbb{R}^{3}$ and, indeed, they frame its fibres. It follows that the functions annihilated by these vector fields are spanned by $x, y, z$ - the coordinates of the homogeneous space $G / S L(3, \mathbb{R})$ in which the curves are immersed. Note that no integration is required ${ }^{6}$. A local coordinate calculation verifies this claim. By step b) we are at liberty to take any one of these as the parameter along the curve. We take $x$ for this purpose. Continuing to follow b) we fix a vector field $\boldsymbol{Z} \in \mathcal{V}$ such that $\boldsymbol{Z} x=1$. At this point we must construct the vector fields that span $\mathcal{V}$. This is straightforward since $\mathcal{V}$ is constructed from the left-invariant vector fields on $G$ (as well as $\left.\partial_{\kappa_{1}}, \partial_{\kappa_{2}}\right)$. We obtain

$$
\begin{aligned}
& v_{1}=a_{1} \partial_{x}+a_{4} \partial_{y}+a_{7} \partial_{z}, \quad v_{2}=a_{2} \partial_{x}+a_{5} \partial_{y}+a_{8} \partial_{z}, \quad v_{3}=a_{3} \partial_{x}+a_{6} \partial_{y}+a_{9} \partial_{z}, \\
& v_{4}=a_{1} \partial_{a_{1}}-a_{3} \partial_{a_{3}}+a_{4} \partial_{a_{4}}-a_{6} \partial_{a_{6}}+a_{7} \partial_{a_{7}}, \quad v_{5}=a_{2} \partial_{a_{1}}+a_{5} \partial_{a_{4}}+a_{8} \partial_{a_{7}}, \\
& v_{6}=a_{3} \partial_{a_{1}}+a_{6} \partial_{a_{4}}+a_{9} \partial_{a_{7}}, \quad v_{7}=a_{1} \partial_{a_{2}}+a_{4} \partial_{a_{5}}+a_{7} \partial_{a_{8}}, \\
& v_{8}=a_{2} \partial_{a_{2}}-a_{3} \partial_{a_{3}}+a_{5} \partial_{a_{5}}-a_{6} \partial_{a_{6}}+a_{8} \partial_{a_{8}}, \quad v_{9}=a_{3} \partial_{a_{2}}+a_{6} \partial_{a_{5}}+a_{9} \partial_{a_{8}}, \\
& v_{10}=a_{1} \partial_{a_{3}}+a_{4} \partial_{a_{6}}, \quad v_{11}=a_{2} \partial_{a_{3}}+a_{5} \partial_{a_{6}}, \quad v_{12}=\partial_{\kappa_{1}}, \quad v_{13}=\partial_{\kappa_{2}},
\end{aligned}
$$

where

$$
a_{9}=\frac{1-a_{2} a_{6} a_{7}+a_{1} a_{6} a_{8}-a_{3} a_{4} a_{8}+a_{3} a_{5} a_{7}}{a_{1} a_{5}-a_{4} a_{2}} .
$$

From these we obtain

$$
\boldsymbol{Z}=\frac{1}{a_{1}}\left(v_{1}+v_{5}+v_{9}+\kappa_{1}\left(v_{7}+3 v_{11}\right)+\kappa_{2} v_{10}\right) \in \mathcal{V} .
$$

Note that $\boldsymbol{Z}$ is the total differential operator in this example. Finally, we let $z_{0}^{1}=y, z_{0}^{2}=z$ as in step c) and compute the higher order coordinates as in step d) by differentiation by $\boldsymbol{Z}$. This construction provides the components of the local equivalence $\phi: G \times \mathbb{R}^{2} \rightarrow J^{5}\left(\mathbb{R}, \mathbb{R}^{2}\right)$ that identifies $\mathcal{V}$ with the contact distribution $\mathcal{C}_{2}^{(5)}$ on $J^{5}\left(\mathbb{R}, \mathbb{R}^{2}\right)$. Local inverse $\psi$ of the map $\phi$ gives the explicit differential invariants $\kappa_{1}, \kappa_{2}$, for curves in $\mathbb{R}^{3}$ up to "equi-affine motions":

$$
\begin{aligned}
\kappa_{1}= & \left(24 z_{2}^{2} w_{3} w_{5}-35 z_{2}^{2} w_{4}^{2}-60 z_{2} w_{3}^{2} z_{4}+60 z_{2} w_{3} z_{3} w_{4}-24 z_{2} w_{2} w_{3} z_{5}+70 z_{2} w_{4} w_{2} z_{4}\right. \\
& \left.-24 z_{2} w_{2} z_{3} w_{5}+60 w_{2} w_{3} z_{3} z_{4}-60 w_{2} z_{3}^{2} w_{4}-35 w_{2}^{2} z_{4}^{2}+24 z_{5} w_{2}^{2} z_{3}\right) /\left(w_{3} z_{2}-w_{2} z_{3}\right)^{7 / 3}, \\
\kappa_{2}= & \left(-18 z_{2}^{3} w_{3} w_{4} w_{5}+25 z_{2}^{3} w_{4}^{3}-36 z_{2}^{2} w_{3}^{3} z_{5}+90 z_{2}^{2} w_{3}^{2} z_{4} w_{4}+36 z_{2}^{2} w_{3}^{2} z_{3} w_{5}\right. \\
& -90 z_{2}^{2} w_{3} z_{3} w_{4}^{2}+18 z_{2}^{2} w_{3} w_{2} z_{4} w_{5}+18 z_{2}^{2} w_{3} w_{2} w_{4} z_{5}-75 z_{2}^{2} w_{2} w_{4}^{2} z_{4}+18 z_{2}^{2} w_{2} z_{3} w_{4} w_{5} \\
& -90 z_{2} w_{3}^{2} w_{2} z_{4}^{2}+72 z_{2} w_{3}^{2} z_{5} w_{2} z_{3}-18 z_{2} w_{3} w_{2}^{2} z_{4} z_{5}-72 z_{2} w_{3} w_{2} z_{3}^{2} w_{5}+90 z_{2} w_{2} z_{3}^{2} w_{4}^{2} \\
& +75 z_{2} w_{2}^{2} w_{4} z_{4}^{2}-18 z_{2} w_{2}^{2} z_{3} w_{4} z_{5}-18 z_{2} w_{2}^{2} z_{3} z_{4} w_{5}+90 w_{3} w_{2}^{2} z_{3} z_{4}^{2}-36 w_{3} z_{5} w_{2}^{2} z_{3}^{2} \\
& \left.-25 w_{2}^{3} z_{4}^{3}-90 w_{2}^{2} z_{3}^{2} w_{4} z_{4}+18 w_{2}^{3} z_{3} z_{4} z_{5}+36 w_{2}^{2} z_{3}^{3} w_{5}\right) /\left(w_{3} z_{2}-w_{2} z_{3}\right)^{7 / 2},
\end{aligned}
$$

where $z_{i}=f^{(i)}(x), w_{j}=g^{(j)}(x)$ for arbitrary smooth functions $f(x), g(x)$ such that

$$
g^{\prime \prime \prime}(x) f^{\prime \prime}(x)-g^{\prime \prime}(x) f^{\prime \prime \prime}(x) \neq 0 .
$$

Thus, if the curve in question has parametrisation $(x, f(x), g(x))$ then the complete set of differential invariants is $\left\{\kappa_{1}, \kappa_{2}\right\}$. Pulling back the remaining nonzero elements of the MaurerCartan form on $G$ by $\psi$ give explicit expressions for invariant differential one-forms on $J^{5}\left(\mathbb{R}, \mathbb{R}^{2}\right)$. One can easily obtain the invariants in an arbitrary parametrisation of the curve or in terms of arclength parametrisation from these formulas but we won't record these here ${ }^{7}$.

\footnotetext{
${ }^{6}$ See Section 5 for further comment on this aspect.

${ }^{7}$ Note that the inversion of $\phi$ is greatly assisted by the fact that the algebraic equations involved are guaranteed, by the proof of Theorem 3.3 in [27] to be block triangular.
} 


\section{Curves in Riemannian manifolds}

The geometries discussed in previous sections of this paper and the one treated in [28] are all of Klein type and one may wonder how the method fares when curvature is introduced. Also, in our previous (illustrative) examples, we have permitted ourselves knowledge of the explicit transitive group action in order to construct the differential system $\mathcal{V}$ and we do not want to make this assumption in general. In this paper we will be content to establish a result for Riemannian geometry and illustrate this by two examples. However, we conjecture that similar results hold for other Cartan geometries.

Let $\omega^{i}, \pi_{j}^{i}$ be the components of the Cartan connection ${ }^{8}$ of an arbitrary Riemannian manifold $(M, g)$, where $\operatorname{dim} M=n$ and $\omega^{i}, 1 \leq i \leq n$ are semi-basic 1-forms for the projection $\mathcal{F}(M) \rightarrow M ; \mathcal{F}(M)$ is the orthonormal frame bundle over $M$. The structure equations for the coframe on $\mathcal{F}(M)$ are

$$
d \omega^{i}=\sum_{j=1}^{n} \omega^{j} \wedge \pi_{j}^{i}, \quad d \pi_{j}^{i}-\sum_{k=1}^{n} \pi_{k}^{i} \wedge \pi_{j}^{k}=\frac{1}{2} \sum_{k, l=1}^{n} R_{j k l}^{i} \omega^{k} \wedge \omega^{l}
$$

where all indices range from 1 to $n$ and $\pi_{j}^{i}+\pi_{i}^{j}=0$ for all $i, j$. Dually, the frame satisfies

$$
\left[\partial_{\omega^{i}}, \partial_{\omega^{j}}\right]=-\frac{1}{2} \sum_{k, l=1}^{n} R_{l i j}^{k} \partial_{\pi_{l}^{k}}, \quad\left[\partial_{\omega^{i}}, \partial_{\pi_{j}^{l}}\right]=-\delta_{i j} \partial_{\omega^{l}}, \quad\left[\partial_{\pi_{l}^{i}}, \partial_{\pi_{k}^{j}}\right]=\delta_{k l} \partial_{\pi_{j}^{i}}+\delta_{i j} \partial_{\pi_{k}^{l}}
$$

We now state the main result of this section.

Theorem 4. Let $n \geq 3$ and $\gamma: I \rightarrow M$ be an immersed curve in an $n$-dimensional Riemannian manifold $(M, g), I \subseteq \mathbb{R}$. Let $\omega^{i}, \pi_{j}^{i}$ be the components of the Cartan connection for $(M, g)$ on the orthonormal frame bundle $\mathcal{F}(M) \rightarrow M$. Then

1. There is a unique integral submanifold of

$$
\begin{aligned}
\mathcal{V}=\{ & \partial_{\omega^{1}}+\kappa_{0}^{1} \partial_{\pi_{1}^{2}}+\kappa_{0}^{2} \partial_{\pi_{2}^{3}}+\cdots+\kappa_{0}^{n-1} \partial_{\pi_{n-1}^{n}}+\sum_{l=1}^{n-2} \kappa_{l}^{1} \partial_{\kappa_{l-1}^{1}} \\
& \left.+\sum_{l=1}^{n-3} \kappa_{l}^{2} \partial_{\kappa_{l-1}}+\sum_{l=1}^{n-4} \kappa_{l}^{3} \partial_{\kappa_{l-1}}+\cdots+\kappa_{1}^{n-2} \partial_{\kappa_{0}^{n-2}}, \partial_{\kappa_{n-2}^{1}}, \partial_{\kappa_{n-3}^{2}}, \ldots, \partial_{\kappa_{0}^{n-1}}\right\},
\end{aligned}
$$

which projects to the Frenet lift of $\gamma$ to the orthonormal frame bundle $\mathcal{F}(M)$.

2. The sub-bundle $\mathcal{V}$ is a uniform Goursat bundle with signature

$$
\langle\overbrace{0,0, \ldots, 0}^{n-1}, n-1\rangle
$$

Hence there is a local diffeomorphism $\phi$ which identifies it with the contact distribution $\mathcal{C}_{n-1}^{(n)}$ on jet space $J^{n}\left(\mathbb{R}, \mathbb{R}^{n-1}\right)$.

3. The local diffeomorphism $\phi$ can be constructed by differentiation and algebraic operations alone.

4. If the isometries of $(M, g)$ act transitively, then the local diffeomorphism $\phi$ induces local coordinate formulas for the complete invariants $\kappa_{0}^{1}, \kappa_{0}^{2}, \ldots, \kappa_{0}^{n-1}$ of the curve $\gamma$ up to isometries of $(M, g)$.

\footnotetext{
${ }^{8}$ See Sharpe [21] for an account of Klein-Cartan geometries and Cartan connections; see also [23, Chapter 7].
} 
Proof. The proofs of 1 and 4 are similar to the proof of Theorem 1. The proof of 2 is complicated to write down in all generality; it is more enlightening to prove it in a sufficiently non-trivial case. We therefore write out the detail proof in the case $n=6$, after which the general case will be clear.

The distribution in question, for $n=6$, is

$$
\begin{aligned}
\mathcal{V}=\left\{\partial_{\omega^{1}}+\sum_{l=1}^{5} \kappa_{0}^{l} \partial_{\pi_{l+1}^{l}}+\sum_{l=1}^{4} \kappa_{l}^{1} \partial_{\kappa_{l-1}^{1}}+\sum_{l=1}^{3} \kappa_{l}^{2} \partial_{\kappa_{l-1}^{2}}+\sum_{l=1}^{2} \kappa_{l}^{3} \partial_{\kappa_{l-1}^{3}}+\kappa_{1}^{4} \partial_{\kappa_{0}^{4}},\right. \\
\left.\partial_{\kappa_{4}^{1}}, \partial_{\kappa_{3}^{2}}, \partial_{\kappa_{2}^{3}}, \partial_{\kappa_{1}^{4}}, \partial_{\kappa_{0}^{5}}\right\} .
\end{aligned}
$$

We are required to prove that $\mathcal{V} \simeq \mathcal{C}_{5}^{(6)}$. Let us arrange some of the frame elements into subsets as follows

$$
\begin{aligned}
& \mathfrak{p}_{1}:\left\{\partial_{\pi_{6}^{5}}\right\}, \quad \mathfrak{p}_{2}:\left\{\partial_{\pi_{5}^{4}}, \partial_{\pi_{6}^{4}}\right\}, \quad \mathfrak{p}_{3}:\left\{\partial_{\pi_{4}^{3}}, \partial_{\pi_{5}^{3}}, \partial_{\pi_{6}^{3}}\right\}, \\
& \mathfrak{p}_{4}:\left\{\partial_{\pi_{3}^{2}}, \partial_{\pi_{4}^{2}}, \partial_{\pi_{5}^{2}}, \partial_{\pi_{6}^{2}}\right\}, \quad \mathfrak{p}_{5}:\left\{\partial_{\pi_{2}^{1}}, \partial_{\pi_{3}^{1}}, \partial_{\pi_{4}^{1}}, \partial_{\pi_{5}^{1}}, \partial_{\pi_{6}^{1}}\right\} .
\end{aligned}
$$

It is easy to show from (12) that

$$
\mathfrak{h}_{\ell}=\bigoplus_{j=1}^{\ell} \mathfrak{p}_{j} \subseteq \mathfrak{s o}(6), \quad 1 \leq \ell \leq 5,
$$

determine a flag of Lie subalgebras. Using this we compute that for each $s$ in the range $1 \leq s \leq 6$, the quotients $\widehat{\mathcal{V}}^{s}:=\mathcal{V}^{(s)} / \mathcal{V}^{(s-1)}$ have basis representatives

$$
\begin{array}{ll}
\widehat{\mathcal{V}}^{(1)}=\left\{\partial_{\kappa_{3}^{1}}, \partial_{\kappa_{2}^{2}}, \partial_{\kappa_{1}^{3}}, \partial_{\kappa_{0}^{4}}, \partial_{\pi_{6}^{5}}\right\}, & \widehat{\mathcal{V}}^{(2)}=\left\{\partial_{\kappa_{2}^{1}}, \partial_{\kappa_{1}^{2}}, \partial_{\kappa_{0}^{3}}, \partial_{\pi_{5}^{4}}, \partial_{\pi_{6}^{4}}\right\}, \\
\widehat{\mathcal{V}}^{(3)}=\left\{\partial_{\kappa_{1}^{1}}, \partial_{\kappa_{0}^{2}}, \partial_{\pi_{4}^{3}}, \partial_{\pi_{5}^{3}}, \partial_{\pi_{6}^{3}}\right\}, & \widehat{\mathcal{V}}^{(4)}=\left\{\partial_{\kappa_{0}^{1}}, \partial_{\pi_{3}^{2}}, \partial_{\pi_{4}^{2}}, \partial_{\pi_{5}^{2}}, \partial_{\pi_{6}^{2}}\right\}, \\
\widehat{\mathcal{V}}^{(5)}=\left\{\partial_{\pi_{2}^{1}}, \partial_{\pi_{3}^{1}}, \partial_{\pi_{4}^{1}}, \partial_{\pi_{5}^{1}}, \partial_{\pi_{6}^{1}}\right\}, & \widehat{\mathcal{V}}^{(6)}=\left\{\partial_{\omega^{2}}, \partial_{\omega^{3}}, \partial_{\omega^{4}}, \partial_{\omega^{5}}, \partial_{\omega^{6}}\right\} .
\end{array}
$$

The Cauchy systems have bases

$\operatorname{Char} \mathcal{V}^{(1)}=\left\{\partial_{\kappa_{4}^{1}}, \partial_{\kappa_{3}^{2}}, \partial_{\kappa_{2}^{3}}, \partial_{\kappa_{1}^{4}}, \partial_{\kappa_{0}^{5}}\right\}$,

Char $\mathcal{V}^{(2)}=\left\{\partial_{\kappa_{3}^{1}}, \partial_{\kappa_{2}^{2}}, \partial_{\kappa_{1}^{3}}, \partial_{\kappa_{0}^{4}}, \partial_{\pi_{6}^{5}}\right\} \oplus \operatorname{Char} \mathcal{V}^{(1)}$,

Char $\mathcal{V}^{(3)}=\left\{\partial_{\kappa_{2}^{1}}, \partial_{\kappa_{1}^{2}}, \partial_{\kappa_{0}^{3}}, \partial_{\pi_{5}^{4}}, \partial_{\pi_{6}^{4}}\right\} \oplus \operatorname{Char} \mathcal{V}^{(2)}$,

Char $\mathcal{V}^{(4)}=\left\{\partial_{\kappa_{1}^{1}}, \partial_{\kappa_{0}^{2}}, \partial_{\pi_{4}^{3}}, \partial_{\pi_{5}^{3}}, \partial_{\pi_{6}^{3}}\right\} \oplus \operatorname{Char} \mathcal{V}^{(3)}$,

Char $\mathcal{V}^{(5)}=\left\{\partial_{\kappa_{0}^{1}}, \partial_{\pi_{3}^{2}}, \partial_{\pi_{4}^{2}}, \partial_{\pi_{5}^{2}}, \partial_{\pi_{6}^{2}}\right\} \oplus \operatorname{Char} \mathcal{V}^{(4)}$

and hence

$$
\mathcal{V}^{(5)} / \operatorname{Char} \mathcal{V}^{(5)}=\left\{\left[\partial_{\omega^{1}}\right],\left[\partial_{\pi_{2}^{1}}\right],\left[\partial_{\pi_{3}^{1}}\right],\left[\partial_{\pi_{4}^{1}}\right],\left[\partial_{\pi_{5}^{1}}\right],\left[\partial_{\pi_{6}^{1}}\right]\right\}
$$

Structure equations (12) show that the singular sub-bundle is

$$
\widehat{\mathcal{B}}=\left\{\left[\partial_{\pi_{2}^{1}}\right],\left[\partial_{\pi_{3}^{1}}\right],\left[\partial_{\pi_{4}^{1}}\right],\left[\partial_{\pi_{5}^{1}}\right],\left[\partial_{\pi_{6}^{1}}\right]\right\}
$$

and therefore the resolvent bundle is given by

$$
\mathcal{R}\left(\mathcal{V}^{(5)}\right)=\left\{\partial_{\pi_{j}^{i}}\right\} \oplus\left\{\partial_{\kappa_{b}^{a}}\right\}=\mathfrak{s o}(6) \oplus \mathbb{R}^{15},
$$


where $a, b, i, j$ range over all possible values. These calculations show that that the derived type of $\mathcal{V}$ is

$$
[[6,0],[11,5],[16,10],[21,15],[26,20],[31,25],[36,36]]
$$

from which one deduce's that the signature of $\mathcal{V}$ is $\langle 0,0,0,0,0,5\rangle$. Since $\mathcal{R}\left(\mathcal{V}^{(5)}\right)$ is integrable and all other hypotheses of Theorem 3 are satisfied with $q=5$ and $k=6$, we have shown that $\mathcal{V}$ is locally equivalent to the contact system $\mathcal{C}_{5}^{(6)}$ on $J^{6}\left(\mathbb{R}, \mathbb{R}^{5}\right)$.

To prove 3, we invoke Theorem 4.2 of [28] which shows that to construct the local equivalence $\phi$ identifying $\mathcal{V}$ with $\mathcal{C}_{5}^{(6)}$ one only requires a complete set of invariants of the resolvent bundle $\mathcal{R}\left(\mathcal{V}^{(5)}\right)$. However, it is elementary to see that no element of $\mathcal{R}\left(\mathcal{V}^{(5)}\right)$ has components tangent to $M$; on the other hand $\mathcal{R}\left(\mathcal{V}^{(5)}\right)$ spans the tangent spaces of the fibres over $M$. Hence, any coordinate system on $M$ provides the needed invariants and no integration need be performed.

In case the Riemannian manifold $M$ does not have a transitive isometry group then $\mathcal{V}$ may simply be regarded as a control system on $M$ with controls $\kappa_{n-2}^{1}, \kappa_{n-3}^{2}, \ldots, \kappa_{0}^{n-1}$ and with all other coordinates on $E$ playing the role of state variables (outputs, in the language of control theory). The arc-length parametrisation along the curve plays the role of time in this control theoretic interpretation. The fact that $\mathcal{V}$ is a Goursat bundle then proves that it is differentially flat, a type of control system currently under investigation in the control community. In fact it is currently a significant open problem in control theory to geometrically characterise all differentially flat control systems. One other way to state Theorem 4 is to say that the "natural" framing of curves in any Riemannian manifold is differentially flat.

If $M$ does possess a transitive isometry group $G$ then the functions $\kappa_{0}^{1}, \ldots, \kappa_{0}^{n-1}$ form a complete set of curve invariants up to the action of $G$.

One may wonder whether Theorem 4 is a genuine advance in the theory and/or practice of moving frames either in the sense of Cartan or in the sense of Fels-Olver. The contention of this paper is that it does represent an advance in both the theory and the practice of moving frames. As to the theory, I argue that endowing curves in a Cartan geometry with an explicit contact structure is significant given the fundamental role that contact structures play in geometry and differential equations. As to the practice of the method of moving frames, in the case of Riemannian manifolds $(M, g)$ we have given a framing for curves in $M$ as solutions of a differential system $\widehat{\Omega}_{\mathfrak{F}}$ for any metric $g$ and shown that it can be explicitly identified with the contact system on a jet space $J^{k}\left(\mathbb{R}, \mathbb{R}^{q}\right)$ for some $k, q$. In case the isometry group of $(M, g)$ acts transitively, this identification delivers the complete set of invariant data including the differential curve invariants. We formulate this construction as an algorithm.

\section{Algorithm Riemannian curves}

INPUT : Riemannian manifold, $(M, g)$, dimension $n \geq 3$.

a) Construct a parametrisation of $S O(n)$ and orthonormal coframe $\omega$ on $M$.

b) Lift $\omega$ to the orthonormal frame bundle over $M$ and build the Cartan connection $\Theta$ for $(M, g)$. This involves linear algebra.

c) From $\Theta$, build the differential system $\mathcal{V}$ defined in Theorem 4 .

d) Apply procedure Contact using, as invariants of the resolvent bundle, any coordinate system on $M$.

e) Construct diffeomorphism $\phi$ by procedure Contact $A$. 
OUTPUT : Local diffeomorphism $\phi$ identifying $\mathcal{V}$ with contact distribution $\mathcal{C}_{n-1}^{(n)}$. In case the isometry group of $(M, g)$ acts transitively, obtain complete, explicit invariant data for curves in $M$, following the inversion of $\phi$.

Note that Riemannian curves is an algorithm in as much as steps a)-e) do not require any integration to be performed. All the steps involved are algebraic.

\subsection{Curves in the Poincaré half-space}

The aim of this subsection is to apply the previous theorem to construct explicit expressions for curvature and torsion for curves in the Poincaré half-space $H^{3}$, with Riemannian metric

$$
g=\frac{d x^{2}+d y^{2}+d z^{2}}{z^{2}}
$$

In principle we could approach this by putting coordinates on the Lie group $S O(3,1)$ and then consider curves in the homogeneous space $S O(3,1) / S O(3)$, as we did in the case of equi-affine space curves. However, this leads to unwieldy expressions which are difficult to handle, even with the help of a computer. Instead, we will use the method of equivalence to construct the Cartan connection for $g$ and then use this to build the canonical Pfaffian system $\Omega_{\mathfrak{F}}$ for the Frenet frame of a generic curve in $H^{3}$. In fact, we will construct the dual vector field distribution $\mathcal{V}=\Omega_{\mathfrak{F}}^{\perp}$

Begin by lifting the 1-forms

$$
\theta^{1}=\frac{d x}{z}, \quad \theta^{2}=\frac{d y}{z}, \quad \theta^{3}=\frac{d z}{z}
$$

to the orthonormal frame bundle $\mathcal{F}\left(H^{3}\right)$ over $H^{3}$ by

$$
\left(\begin{array}{l}
\omega^{1} \\
\omega^{2} \\
\omega^{3}
\end{array}\right)=O\left(\begin{array}{l}
\theta^{1} \\
\theta^{2} \\
\theta^{3}
\end{array}\right)
$$

where

$$
O=\left(\begin{array}{ccc}
\cos b \cos c-\sin a \sin b \sin c & -\cos a \sin c & \sin a \cos b \sin c+\sin b \cos c \\
\cos b \sin c+\sin a \sin b \cos c & \cos a \cos c & \sin b \sin c-\sin a \cos b \cos c \\
-\cos a \sin b & \sin a & \cos a \cos b
\end{array}\right)
$$

parametrises $S O(3)$. The fibres of the orthonormal frame bundle $\mathcal{F}\left(H^{3}\right) \rightarrow H^{3}$ are diffeomorphic to $S O(3)$ whose Maurer-Cartan form is the $\mathfrak{s o}(3)$-valued 1-form

$$
\Omega=\left(\begin{array}{ccc}
0 & \omega_{2}^{1} & \omega_{3}^{1} \\
\omega_{1}^{2} & 0 & \omega_{3}^{2} \\
\omega_{1}^{3} & \omega_{2}^{3} & 0
\end{array}\right)
$$

where

$$
\omega_{2}^{1}=-d c-\sin a d b, \quad \omega_{3}^{1}=\sin c d a+\cos a \cos c d b, \quad \omega_{3}^{2}=\sin c \cos a d b-\cos c d a,
$$

and $\omega_{i}^{j}+\omega_{j}^{i}=0$. As is usual in the method of equivalence, we compute the structure equations of the semi-basic forms obtaining

$$
d \omega^{1}=\omega_{2}^{1} \wedge \omega^{2}+\omega_{3}^{1} \wedge \omega^{3}+(\sin c \sin b-\cos c \sin a \cos b) \omega^{1} \wedge \omega^{2}+\cos a \cos b \omega^{1} \wedge \omega^{3},
$$




$$
\begin{aligned}
d \omega^{2}= & \omega_{1}^{2} \wedge \omega^{1}+\omega_{3}^{2} \wedge \omega^{3}-(\cos c \sin b+\sin a \cos b \sin c) \omega^{1} \wedge \omega^{2}+\cos a \cos b \omega^{2} \wedge \omega^{3}, \\
d \omega^{3}= & \omega_{1}^{3} \wedge \omega^{1}+\omega_{2}^{3} \wedge \omega^{2}-(\sin b \cos c+\sin a \cos b \sin c) \omega^{1} \wedge \omega^{3} \\
& -(\sin b \sin c-\sin a \cos b \cos c) \omega^{2} \wedge \omega^{3} .
\end{aligned}
$$

All torsion can be absorbed by redefining connection forms

$$
\begin{aligned}
& \pi_{2}^{1}=\omega_{2}^{1}+(\sin c \sin b-\sin a \cos b \cos c) \omega^{1}-(\sin a \cos b \sin c+\sin b \cos c) \omega^{2}, \\
& \pi_{3}^{1}=\omega_{3}^{1}+\cos a \cos b \omega^{1}-(\sin a \cos b \sin c+\sin b \cos c) \omega^{3} \\
& \pi_{3}^{2}=\omega_{3}^{2}+\cos a \cos b \omega^{2}-(\sin b \sin c-\sin a \cos b \cos c) \omega^{3}
\end{aligned}
$$

and we obtain structure equations

$$
d \omega^{1}=\pi_{2}^{1} \wedge \omega^{2}+\pi_{3}^{1} \wedge \omega^{3}, \quad d \omega^{2}=-\pi_{2}^{1} \wedge \omega^{1}+\pi_{3}^{2} \wedge \omega^{3}, \quad d \omega^{3}=-\pi_{3}^{1} \wedge \omega^{1}-\pi_{3}^{2} \wedge \omega^{2} .
$$

The remaining structure equations are

$$
d \pi_{2}^{1}=-\pi_{3}^{1} \wedge \pi_{3}^{2}+\omega^{1} \wedge \omega^{2}, \quad d \pi_{3}^{1}=\pi_{2}^{1} \wedge \pi_{3}^{2}+\omega^{1} \wedge \omega^{3}, \quad d \pi_{3}^{2}=-\pi_{2}^{1} \wedge \pi_{3}^{1}+\omega^{2} \wedge \omega^{3} .
$$

These structure equations are those of the Maurer-Cartan form on $S O(3,1)$. Moreover, we are now able to define the $\mathfrak{e}(3)$-valued Cartan connection

$$
\widehat{\Omega}=\left(\begin{array}{cccc}
0 & 0 & 0 & 0 \\
\omega^{1} & 0 & \pi_{2}^{1} & \pi_{3}^{1} \\
\omega^{2} & \pi_{1}^{2} & 0 & \pi_{3}^{2} \\
\omega^{3} & \pi_{1}^{3} & \pi_{2}^{3} & 0
\end{array}\right)
$$

with curvature

$$
d \widehat{\Omega}+\widehat{\Omega} \wedge \widehat{\Omega}=\left(\begin{array}{cccc}
0 & 0 & 0 & 0 \\
0 & 0 & \omega^{1} \wedge \omega^{2} & \omega^{1} \wedge \omega^{3} \\
0 & \omega^{2} \wedge \omega^{1} & 0 & \omega^{2} \wedge \omega^{3} \\
0 & \omega^{3} \wedge \omega^{1} & \omega^{3} \wedge \omega^{2} & 0
\end{array}\right)
$$

for metric $g$.

Setting $n=3$ in (13), and adopting the usual notation $\kappa_{0}^{1}=\kappa, \kappa_{0}^{2}=\tau, \kappa_{1}^{1}=\kappa_{1}$, we study the integral submanifolds of

$$
\mathcal{V}=\left\{\partial_{\omega^{1}}+\kappa \partial_{\pi_{2}^{1}}+\tau \partial_{\pi_{3}^{2}}+\kappa_{1} \partial_{\kappa}, \partial_{\kappa_{1}}, \partial_{\tau}\right\}
$$

It is easy to check that $\mathcal{V}$ has derived type

$$
[[3,0],[5,2],[7,4],[9,9]] \text {. }
$$

It can be checked that $\rho_{1}=\rho_{2}=0$ and hence the signature of $\mathcal{V}$ is $\langle 0,0,2\rangle$. This is the signature of contact system $\mathcal{C}_{2}^{(3)}$. To complete the check that $\mathcal{V}$ is diffeomorphic to $\mathcal{C}_{2}^{(3)}$, we compute the resolvent bundle and check its integrability.

We find that Char $\mathcal{V}^{(2)}=\left\{\partial_{\pi_{3}^{2}}, \partial_{\kappa}, \partial_{\kappa_{1}}, \partial_{\tau}\right\}$, and

$$
\mathcal{V}^{(2)} / \operatorname{Char} \mathcal{V}^{(2)}=\left\{\left[\partial_{\omega^{1}}\right],\left[\partial_{\pi_{2}^{1}}\right],\left[\partial_{\pi_{3}^{1}}\right]\right\},
$$

whose structure is

$$
\left[\partial_{\omega^{1}}, \partial_{\pi_{2}^{1}}\right] \equiv \partial_{\omega^{2}}, \quad\left[\partial_{\omega^{1}}, \partial_{\pi_{3}^{1}}\right] \equiv \partial_{\omega^{3}}, \quad\left[\partial_{\pi_{2}^{1}}, \partial_{\pi_{3}^{1}}\right] \equiv 0 \quad \bmod \text { Char } \mathcal{V}^{(2)}
$$


Hence, the singular bundle is $\widehat{\mathcal{B}}=\left\{\left[\partial_{\pi_{2}^{1}}\right],\left[\partial_{\pi_{3}^{1}}\right]\right\}$ and consequently the resolvent bundle is

$$
\mathcal{R}\left(\mathcal{V}^{(2)}\right)=\left\{\partial_{\pi_{2}^{1}}, \partial_{\pi_{3}^{1}}, \partial_{\pi_{3}^{2}}, \partial_{\kappa}, \partial_{\kappa_{1}}, \partial_{\tau}\right\}=\mathfrak{s o}(3) \oplus \mathbb{R}^{3}
$$

which is clearly integrable. An easy calculation in local coordinates verifies that the invariants of $\mathcal{R}\left(\mathcal{V}^{(2)}\right)$ are indeed the coordinates $x, y, z$ on the base of the orthonormal frame bundle $\mathcal{F}\left(H^{3}\right) \rightarrow H^{3}$.

According to the Theorem we take one of $x, y, z$ as the independent variable. If $y$ is taken for this purpose, then we can take $\mathcal{D}=\partial_{\omega^{1}}+\kappa \partial_{\pi_{2}^{1}}+\tau \partial_{\pi_{3}^{2}}+\kappa_{1} \partial_{\kappa}$ as the direction of the total differential operator since $0 \neq \mathcal{D}(y)=-z \cos a \sin c=\mu$. Then the operator of total differentiation is

$$
\boldsymbol{Z}=\mu^{-1}\left(\partial_{\omega^{1}}+\kappa \partial_{\pi_{2}^{1}}+\tau \partial_{\pi_{3}^{2}}+\kappa_{1} \partial_{\kappa}\right)
$$

Setting $u=x, v=z$, the remaining contact coordinates for $\mathcal{V}$ are then provided by differentiation

$$
u_{1}=\boldsymbol{Z} u, \quad v_{1}=\boldsymbol{Z} v, \quad u_{2}=\boldsymbol{Z} u_{1}, \quad v_{2}=\boldsymbol{Z} v_{1}, \quad u_{3}=\boldsymbol{Z} u_{2}, \quad v_{3}=\boldsymbol{Z} v_{2} .
$$

The map $\phi$

$$
\left(x, y, z, a, b, c, \kappa, \kappa_{1}, \tau\right) \mapsto\left(y, u, v, u_{1}, v_{1}, u_{2}, v_{2}, u_{3}, v_{3}\right)
$$

pushes $\mathcal{V}$ forward to $\mathcal{C}_{2}^{(3)}$, which, in contact coordinates has the form

$$
\left\{\partial_{y}+u_{1} \partial_{u}+v_{1} \partial_{v}+u_{2} \partial_{u_{1}}+v_{2} \partial_{v_{1}}+u_{3} \partial_{u_{2}}+v_{3} \partial_{v_{2}}, \partial_{u_{3}}, \partial_{v_{3}}\right\}
$$

A local inverse of $\phi$ is easily constructed and provides all the invariant data for curves in $H^{3}$. In particular we deduce explicit expressions for curvature and torsion for curves $\gamma(y)=(u(y), y, v(y))$, where for instance $u_{1}, v_{2}$, denote derivatives $d u / d y, d^{2} v / d y^{2}$, etc. We obtain

$$
\begin{aligned}
\kappa= & -\left(u_{1}^{6}+2 u_{1}^{4} v_{1}^{2}+2 u_{1}^{4} v v_{2}+3 u_{1}^{4}-2 u_{1}^{3} v u_{2} v_{1}+v_{1}^{4} u_{1}^{2}+2 v_{1}^{2} u_{1}^{2} v v_{2}+4 u_{1}^{2} v v_{2}+u_{1}^{2} v^{2} v_{2}^{2}\right. \\
& +4 v_{1}^{2} u_{1}^{2}+3 u_{1}^{2}-2 u_{1} v^{2} u_{2} v_{1} v_{2}-2 u_{1} v u_{2} v_{1}^{3}-2 u_{1} v_{1} u_{2} v+1+v_{1}^{4}+v^{2} u_{2}^{2} v_{1}^{2}+2 v_{1}^{2} v v_{2} \\
& \left.+2 v v_{2}+v_{2}^{2} v^{2}+v^{2} u_{2}^{2}+2 v_{1}^{2}\right)^{1 / 2} /\left(v_{1}^{2}+u_{2}^{2}+1\right)^{3 / 2}, \\
\tau= & \left(3 u_{2}^{2} u_{1}+3 u_{2} v_{1} v_{2}+v u_{2} v_{3}-u_{3} u_{1}^{2}-u_{3} v_{1}^{2}-u_{3} v v_{2}-u_{3}\right) v^{2} /\left(u_{2}^{2} v^{2} v_{1}^{2}+u_{2}^{2} v^{2}\right. \\
& -2 u_{2} v^{2} v_{1} u_{1} v_{2}-2 u_{2} v v_{1} u_{1}-2 v_{1}^{3} u_{1} u_{2} v-2 u_{1}^{3} v_{1} u_{2} v+u_{1}^{2} v^{2} v_{2}^{2}+v^{2} v_{2}^{2}+4 u_{1}^{2} v v_{2} \\
& +2 v_{1}^{2} v v_{2}+2 v v_{2}+2 u_{1}^{4} v v_{2}+2 v_{1}^{2} u_{1}^{2} v v_{2}+1+2 v_{1}^{2}+v_{1}^{4}+4 u_{1}^{2} v_{1}^{2}+3 u_{1}^{2}+2 u_{1}^{4} v_{1}^{2} \\
& \left.+v_{1}^{4} u_{1}^{2}+3 u_{1}^{4}+u_{1}^{6}\right) .
\end{aligned}
$$

Once again, the algebraic system that is presented for solution in this task is guaranteed to have a block triangular structure.

We remark that semi-circular arcs parallel to the $y-z$ plane

$$
\gamma(y)=\left(C_{1}, y, \sqrt{C_{2}^{2}-y^{2}}\right), \quad C_{2}>0, \quad-C_{2}<y<C_{2}
$$

are geodesics in $H^{3}$; accordingly it can be checked that $\kappa(\gamma(y))=\tau(\gamma(y)) \equiv 0$. 


\subsection{Curves in constant curvature Riemannian 3-manifolds}

Let $\lambda$ be any nonzero real number. Here we point out that the construction of the previous subsection can be carried out for the three dimensional Riemannian manifold $\left(M(\lambda), g_{\lambda}\right)$ with metric

$$
g_{\lambda}=\frac{d x^{2}+d y^{2}+d z^{2}}{\left(1+\frac{\lambda}{4}\left(x^{2}+y^{2}+z^{2}\right)\right)^{2}}
$$

of constant curvature $\lambda$, where $M(\lambda)$ is an open subset of $\mathbb{R}^{3}$. Exactly the same calculation as before but with

$$
\theta^{1}=\frac{d x}{\Lambda}, \quad \theta^{2}=\frac{d y}{\Lambda}, \quad \theta^{3}=\frac{d z}{\Lambda},
$$

where $\Lambda=1+\frac{\lambda}{4}\left(x^{2}+y^{2}+z^{2}\right)$ gives rise to the corresponding Cartan connection

$$
\widehat{\Omega}=\left(\begin{array}{cccc}
0 & 0 & 0 & 0 \\
\omega^{1} & 0 & \pi_{2}^{1} & \pi_{3}^{1} \\
\omega^{2} & \pi_{1}^{2} & 0 & \pi_{3}^{2} \\
\omega^{3} & \pi_{1}^{3} & \pi_{2}^{3} & 0
\end{array}\right)
$$

with curvature

$$
d \widehat{\Omega}+\widehat{\Omega} \wedge \widehat{\Omega}=-\lambda\left(\begin{array}{cccc}
0 & 0 & 0 & 0 \\
0 & 0 & \omega^{1} \wedge \omega^{2} & \omega^{1} \wedge \omega^{3} \\
0 & \omega^{2} \wedge \omega^{1} & 0 & \omega^{2} \wedge \omega^{3} \\
0 & \omega^{3} \wedge \omega^{1} & \omega^{3} \wedge \omega^{2} & 0
\end{array}\right)
$$

for the metric $g_{\lambda}$. Exactly the same calculation as the one carried out for the Poincaré half-space gives rise to the curvature and torsion for curves in $\left(M(\lambda), g_{\lambda}\right)$. We get

$$
\begin{aligned}
\kappa_{\lambda} & =\lambda \frac{\sqrt{\left(y u_{1}-u\right)^{2}+\left(y v_{1}-v\right)^{2}+\left(v u_{1}-u v_{1}\right)^{2}}}{2 \sqrt{u_{1}^{2}+v_{1}^{2}+1}} \\
\tau_{\lambda} & =-\frac{\left(4+\lambda\left(y^{2}+v^{2}+u^{2}\right)\right)^{2}\left(u v_{3}-v u_{3}+y\left(v_{1} u_{3}-v_{3} u_{1}\right)\right)}{8 \lambda\left(1+u_{1}^{2}+v_{1}^{2}\right)\left(\left(y u_{1}-u\right)^{2}+\left(y v_{1}-v\right)^{2}+\left(v u_{1}-u v_{1}\right)^{2}\right)} .
\end{aligned}
$$

The point to note here as in the previous example is that we are not required to know the explicit formulas for the action of the isometries on $M(\lambda)$ before the invariants and the moving frame can be computed. Only infinitesimal data is required, in the form of the Cartan connection and then no integration need be performed.

Remark 4. Interestingly, $\tau_{\lambda}$ is not a continuous function of $\lambda$ at $\lambda=0$, while $\lim _{\lambda \rightarrow 0} \kappa_{\lambda}$ is not the curvature of the curve in the corresponding limiting metric $\lim _{\lambda \rightarrow 0} g_{\lambda}$, which is Euclidean.

\section{Closing remarks}

In this paper we have demonstrated that curves in various geometries can be endowed with a contact geometry by combining Cartan's classical construction of moving frames with the generalised Goursat normal form. In particular we have shown that curves in any Riemannian manifold can be endowed with a contact geometry regardless of the nature of its isometry group or curvature tensor. 
We have also been concerned with the problem of explicitly computing differential invariants of curves immersed in spaces equipped with a transitive action of a Lie group. If this Lie group action is explicitly known and not too complicated then the method of choice for computing the differential invariants and other geometric data is the normalisation of the group action as in the equivariant moving frames method of Fels and Olver, described in [9, 10]. This method is very general, has a simple and elegant theoretical foundation and presents as simple a computational task as could be hoped for. However, if the explicit group action is not known or it is known but too complicated to work with and if the goal is explicit expressions for differential invariants then the Fels-Olver method can't readily be used to compute invariants explicitly ${ }^{9}$. In this case, we have shown that for Riemannian manifolds $(M, g)$, the contact geometry can be fruitfully used to derive differential invariants and this requires as input data only the metric $g$ and a realisation of the structure group $S O(n)$. With this data the Cartan connection for $(M, g)$ can be constructed by linear algebra and differentiation. Subsequently, Theorem 4 provides an algorithm, Riemannian curves, for the curve invariants and, if required the Fels-Olver equivariant moving frame.

The symbolic computational aspects of procedure Contact and algorithm Riemannian curves presented in this paper should be mentioned briefly. The Maple package DifferentialGeometry is ideally suited to the computation of all the relevant bundles and determining the derived type of any sub-bundle $\mathcal{V} \subset T M$ over manifold $M$. For instance, the two Riemannian examples presented in Section 5, take only a few minutes to complete commencing only with the metric and realisation of matrix group $S O(3)$. Furthermore, the construction of differential curve invariants requires the inverse of the local diffeomorphism $\phi$ produced by procedure Contact. The proof of correctness of Contact in [28] shows that this algebraic problem will be block triangular. Thus the procedures discussed in this paper have quite good computational features.

However, of much greater significance stands the proposition that the Frenet frames along a curve and hence the curve itself can be endowed with a contact geometry. This should have significance not only for the geometry of curves but also for Cartan's method of moving frames as well as for the equivariant moving frames method of Fels and Olver. This is because contact systems are fundamental geometric objects and play a central role in differential geometry and differential equations. In fact, the construction of any contact system out of the components of a Cartan connection can in many ways replace or complement the step by step construction of moving frames championed by Cartan. What is more, a characterisation of contact systems in arbitrary jet spaces in the spirit of the Goursat normal form is known [2, 30] and could be applied to study the geometry of submanifolds of dimension $p>1$ in general geometries as we have done here in the case $p=1$. This raises the interesting question of the extent to which the results of this paper can be extended to homogeneous spaces in general and how they are connected to existing theory such as $[5,6,8,12,14,11,26,1,21,9,10,24]$.

In this respect it should be mentioned that the Fels-Olver theory of moving frames has application well beyond the explicit calculation of differential invariants and moving frames. Much can be accomplished within the theory even without this explicit knowledge; see Mansfield [16] for details. What we hope to have achieved in this paper is the presention of evidence supporting the proposition that it is useful to enrich the philosophy and practice of moving frames by exploring its links with contact geometry.

Finally, we mention that an intriguing question is the relationship between the contact geometry of curves as explained here and the integrable motion of curves in various ambient manifolds $[13,7,17,19]$.

\footnotetext{
${ }^{9}$ However, it is sometimes possible to simplify the task of normalising the group action by using the known action of a subgroup, a result due to Kogan, [15].
} 


\section{Acknowledgements}

I am indebted to the anonymous referees for insightful comments and for corrections which greatly improved the paper. Any remaining errors are mine.

\section{References}

[1] Alekseev D.V., Vinogradov A.M., Lychagin V.V., Geometry I, Encycl. Math. Sci., Vol. 28, Spinger-Verlag, Berlin, 1991.

[2] Bryant R.L., Some aspects of the local and global theory of Pfaffian systems, PhD thesis, University of North Carolina, Chapel-Hill, 1979.

[3] Bryant R.L., Chern S.S., Gardner R.B., Goldschmidt H.L., Griffiths P.A., Exterior differential systems, Mathematical Sciences Research Institute Publications, Vol. 18, Springer-Verlag, New York, 1991.

[4] Cartan É., La méthode du repère mobile, la théorie des groupes continus et les espaces généralisés Hermann \& Cie, Paris, 1935.

[5] Cartan É., La théorie des groupes finis et continus et la géométrie différentielle traitees par la méthode du repère mobile, Gautier-Villars, Paris, 1937.

[6] Chern S.S., Moving frames, in The Mathematical Heritage of Élie Cartan (Lyon, 1984), Astérique, Vol. 1985, Numero Hors Serie, 1985, 67-77.

[7] Chou K.-S., Qu C.-Z., Integrable equations arising from motions of plane curves, Phys. D 162 (2002), 9-33.

[8] Favard J., Cours de géométrie différentielle locale, Gauthier-Villars, Paris, 1957.

[9] Fels M., Olver P.J., Moving coframes. I. A practical algorithm, Acta Appl. Math. 51 (1998), 161-213.

[10] Fels M., Olver P.J., Moving coframes. II. Regularization and theoretical foundations, Acta Appl. Math. 55 (1999), 127-208.

[11] Green M.L., The moving frame, differential invariants and rigidity theorems for curves in homogeneous spaces, Duke Math. J. 45 (1978), 735-779.

[12] Griffiths P.A., On Cartan's method of Lie groups and moving frames as applied to uniqueness and existence questions in differential geometry, Duke Math. J. 41 (1974), 775-814.

[13] Ivey T., Integrable geometric evolution equations for curves, in The Geometrical Study of Differential Equations (Washington, DC, 2000), Contemp. Math., Vol. 285, Amer. Math. Soc., Providence, RI, 2001, $71-84$.

[14] Jensen G.R., Higher order contact of submanifolds of homogeneous spaces, Lecture Notes in Mathematics, Vol. 610, Springer-Verlag, Berlin - New York, 1977.

[15] Kogan I.A., Inductive approach to moving frames and applications in classical invariant theory, PhD Thesis, University of Minesota, 2000.

[16] Mansfield E.L., A guide to the symbolic invariant calculus, Cambridge University Press, Cambridge, to appear.

[17] Mansfield E.L., van der Kamp P.E., Evolution of curve invariants and lifting integrability, J. Geom. Phys. 56 (2006), 1294-1325.

[18] Olver P.J., Moving frames - in geometry, algebra, computer vision and numerical analysis, in Foundations of Computational Mathematics (Oxford, 1999), Editors R. DeVore, A. Iserles and E. Süli, London Math. Soc. Lecture Note Ser., Vol. 284, Cambridge University Press, Cambridge, 2001, 267-297.

[19] Olver P.J., Invariant submanifold flows, J. Phys. A: Math. Theor. 41 (2008), 344017, 22 pages.

[20] Shadwick W.F., Sluis W.M., Dynamic feedback for the classical geometries, in Differential Geometry and Mathematical Physics (Vancouver, BC, 1993), Contemp. Math., Vol. 170, Amer. Math. Soc., Providence, RI, 1994, 207-213.

[21] Sharpe R., Differential geometry. Cartan's generalisation of Klein's erlangen program, Graduate Texts in Mathematics, Springer-Verlag, New York, 1997.

[22] Spivak M., A comprehensive introduction to differential geometry, Vol. 1, Publish or Perish Press, 1970.

[23] Spivak M., A comprehensive introduction to differential geometry, Vol. 2, Publish or Perish Press, 1979.

[24] Streltsova I.S., R-conformal invariants of curves, Izv. Vyssh. Uchebn. Zaved. Mat. 53 (2009), no. 5, 67-69. 
[25] Stormark O., Lie's structural approach to PDE systems, Encyclopedia of Mathematics and its Applications, Vol. 80, Cambridge University Press, Cambridge, 2000.

[26] Sulanke R., On É. Cartan's method of moving frames, Colloq. Math. Soc. Janos Bolyai, Vol. 31, Differential Geometry, Budapest, 1979.

[27] Vassiliou P., A constructive generalised Goursat normal form, Differential Geom. Appl. 24 (2006), 332-350, math.DG/0404377.

[28] Vassiliou P., Efficient construction of contact coordinates for partial prolongations, Found. Comput. Math. 6 (2006), 269-308, math.DG/0406234.

[29] Vessiot E., Sur l'intégration des faisceaux de transformations infinitésimales dans le cas où, le degré du faisceau étant $n$, celui du faisceau derivée est $n+1$, Ann. Sci. École Norm. Sup. (3) 45 (1928), 189-253.

[30] Yamaguchi K., Contact geometry of higher order, Japan. J. Math. (N.S.) 8 (1982), 109-176.

Yamaguchi K., Geometrization of jet bundles, Hokkaido Math. J. 12 (1983), 27-40. 